\title{
Co-construction et expérimentation d'une bande dessinée numérique pour la classe : les Grandiloquents, épisode sur la gravitation
}

Valentin Maron, Laurence Bordenave et Barbara Govin

\section{(2) OpenEdition \\ Journals \\ Édition électronique \\ URL : http://journals.openedition.org/trema/5215 \\ DOI : 10.4000/trema.5215 \\ ISSN : 2107-0997 \\ Éditeur \\ Faculté d'Éducation de l'université de Montpellier}

\section{Édition imprimée}

ISBN : 979-10-96627-07-3

ISSN : 1167-315X

Référence électronique

Valentin Maron, Laurence Bordenave et Barbara Govin, « Co-construction et expérimentation d'une bande dessinée numérique pour la classe : les Grandiloquents, épisode sur la gravitation », Tréma [En ligne], 51 | 2019, mis en ligne le 01 avril 2019, consulté le 09 août 2019. URL : http:// journals.openedition.org/trema/5215; DOI : 10.4000/trema.5215

Ce document a été généré automatiquement le 9 août 2019.

Trema 


\title{
Co-construction et expérimentation d'une bande dessinée numérique pour la classe : les Grandiloquents, épisode sur la gravitation
}

\author{
Valentin Maron, Laurence Bordenave et Barbara Govin
}

\section{Introduction}

1 Les auteurs de bande dessinée s'emparent régulièrement des savoirs scientifiques pour construire des récits où la science, matière à conter, est au cœur de la narration visuelle. Écrits pour le grand public, ces ouvrages ont une portée informative variable selon les intentions médiatiques et artistiques des auteurs (Bordenave, 2016). Dans l'ensemble, les premières narrations graphiques scientifiques sont souvent explicatives, au sens où elles tentent de rendre accessible au lecteur des concepts, des phénomènes à la manière d'un ouvrage «scientifique ». C'est le cas par exemple des séries The Cartoon History of the Universe (1977), Les Aventures d'Anselme Lanturlu (1979) ou Manga Sciences (1991). D'autres collections de bandes dessinées plus récentes s'inspirent des codes du reportage pour donner au discours une couleur résolument informative, telle que l'œuvre de Marion Montaigne. Certains documentaires graphiques affichent quant à eux une ambition surtout créative (Raux, 2018), notamment par l'usage de métaphores visuelles figurant des concepts scientifiques. Ces bandes dessinées sont fréquemment le fruit de collaborations étroites au sein d'un duo scientifique-artiste, comme c'est le cas pour les tomes scientifiques de La Petite Bédéthèque des Savoirs. Des entretiens menés par Collver et Weitkamp (2018) auprès d'auteurs anglophones de bandes dessinées scientifiques montrent que la plupart d'entre eux souhaitent divertir autant qu'informer, quelle que soit le type de narration empruntée.

2 L'évaluation de l'impact de ces bandes dessinées sur l'acquisition des informations mises à disposition dans ces récits reste en revanche peu étudiée (Farinella, 2018; Tatalovic, 
2009), et lorsqu'elle l'est, c'est principalement dans le contexte scolaire (primaire, secondaire et supérieur). Des recherches se sont ainsi intéressées à la façon dont la lecture de bandes dessinées scientifiques pouvaient transformer l'attitude vis-à-vis de la discipline traitée (Hosler \& Boomer, 2011) ou déclencher des démarches d'investigation (Arguel et al., 2017; Blanquet, 2016);

En outre, les expérimentations menées en classe s'appuient parfois sur des ouvrages de bande dessinée générale qui n'ont pas pour objet spécifique de transmettre des savoirs scientifiques, tels que Tintin (Chevalier, 2013 ; Durocher, 2016), Astérix ou Le Marsupilami (Blanquet, 2016). Dans ce cas, il s'agit de détourner l'intention initiale des auteurs en exploitant une vignette, une planche ou une situation d'une bande dessinée "classique " à des fins pédagogiques.

4 Des auteurs de BD de l'association Stimuli, spécialisée dans les liens entre bande dessinée et la transmission des sciences, et des chercheurs du Laboratoire de didactique André Revuz de l'Université Paris Diderot se sont associés pour concevoir et analyser, en collaboration, une série de bandes dessinées numériques pour le collège, et plus précisément le cycle 4 (5e, 4e, 3e). Actuellement en cours de développement cette série se nomme Les Grandiloquents. Dans ce projet, les chercheurs en didactique sont invités à participer activement au choix des thèmes, à la relecture, à l'amélioration du scénario et du storyboard de chaque épisode, et conduisent des expérimentations en classe de la série.

5 La série est prévue pour être composée d'épisodes de bande dessinée écrits et réalisés pour une lecture sur écran. La bande dessinée est donc ici conçue pour un fonctionnement rhétorique selon la typologie de Benoit Peeters (2002), où les éléments formels (ici l'image et son apparition/disparition à l'écran) ont pour principale fonction de servir le récit. Les récits sont inspirés de l'histoire des sciences et ont pour principal objectif de faire émerger les conceptions initiales des élèves et de susciter le débat en classe. Un premier épisode pilote portant sur la circulation sanguine (Episode $n^{\circ} 1$ ) a déjà été expérimenté auprès de 4 classes de cinquième. L'évaluation a montré une diversité des pratiques enseignantes s'appuyant sur le récit graphique pour inviter les élèves à problématiser sur la circulation sanguine (Pelé, 2016; Bordenave, Crépin-Obert et Pelé, 2016). L'expérimentation de cet épisode $n^{\circ} 1$ a également permis de souligner que la longueur - et par conséquent le temps de lecture - de l'épisode était trop grande, et que le positionnement du problème mis en scène devait être plus explicite.

6 L'objet du présent travail est de discuter la co-construction et l'expérimentation d'un nouvel épisode sur le thème de la gravitation (Episode $n^{\circ} 2$ ), inspiré d'une recherche en didactique sur ce sujet (Maron, 2015). Dans la première partie seront présentés le contexte de la série, les fondements de l'épisode, et la logique du cheminement conceptuel proposé afin de mettre en avant l'articulation entre les enjeux didactiques et les particularités du récit en bande dessinée. Nous présenterons dans une deuxième partie la méthodologie d'expérimentation de l'épisode, une synthèse des résultats, et les pistes d'amélioration qui en découlent pour l'épisode et son utilisation. 


\section{Co-construction d'un épisode}

\section{Principes narratif et graphique de la série}

7 La narration de chaque épisode de la série est fondée sur la mise en scène de quatre adolescents membres d'un club de théatre amenés à jouer des pièces faisant écho à l'histoire de la thématique scientifique traitée. A titre d'exemple, l'épisode $\mathrm{n}^{\circ} 1$ sur la circulation sanguine met en scène les Grandiloquents (Diane, Iris, Titouan et Tom) en prise avec "Le Malade Imaginaire" de Molière, pièce où la controverse entre "irrigateurs" et “circulateurs" est suggérée par le dramaturge (Pelé, 2016).

8 L'objectif de ce choix narratif est double. Il invite les élèves à s'identifier aux personnages de la série par l'entremise des situations suscitées par le jeu théâtral d'adolescents de leur âge, et par conséquent à se confronter également aux questionnements auxquels les Grandiloquents doivent répondre au cours du récit. L'approche historique des savoirs est susceptible d'aider les élèves à la compréhension des obstacles épistémologiques rencontrés par les scientifiques dans le passé, par analogie avec les leurs, ainsi qu'à explorer les franchissements réalisés par ces scientifiques au cours de l'élaboration de leurs concepts, lois ou théories (Audigier et Fillon, 1991). De fait, le récit de bande dessinée est ici pensé pour interroger les élèves sur leurs propres conceptions et les guider dans la résolution des questionnements soulevés, avec l'aide de leur professeur.

$\mathrm{Du}$ point de vue graphique, les représentations des concepts scientifiques, lorsqu'elles sont nécessaires, sont inspirées principalement des représentations historiques (les planches anatomiques de Vésale au XVIème siècle dans l'épisode $n^{\circ} 1$, une illustration de Newton sur la gravitation dans l'épisode $n^{\circ} 2$ ). Toute représentation graphique des savoirs scientifiques telle que les schémas explicatifs est évitée pour ne pas empiéter sur la séquence pédagogique développée par l'enseignant. Par ailleurs, le parti-pris des auteurs est d'explorer d'autres mises en scène des contenus par la bande dessinée : par exemple, des mises en situations analogues entre le monde fictionnel et le monde réel permettant de visualiser une expérience de pensée, ainsi que des dialogues dans lesquels les élèves peuvent projeter leurs propres représentations d'un concept (de Hosson, 2016).

\section{Les fondements de l'épisode étudié}

Plusieurs éléments interdépendants ont influencé l'écriture du récit de l'épisode $\mathrm{n}^{\circ} 2$ sur la gravitation: les objectifs d'apprentissage du programme de physique-chimie pour le cycle $4^{1}$, l'état de l'art sur la recherche en didactique autour de la notion de gravitation, les principes iconico-narratifs de la série présentés en amont, la navigation numérique dans le récit et la pièce de théâtre choisie comme prétexte au récit.

D'après la littérature en didactique sur la gravitation, l'obstacle majeur pour comprendre cette notion est l'idée que la gravité ou gravitation ne s'applique pas dans l'espace (Kavanagh \& Sneider, 2006). L'objectif général défini en conséquence est alors de proposer un récit graphique permettant de montrer en quoi le mouvement orbital dans l'espace peut être relié au mouvement de chute libre sur Terre et ainsi à la notion de gravité.

12 Le développement multimédia choisi pour la bande dessinée finale tel que défini pour l'épisode $n^{\circ} 1$ (Bordenave, 2016) est basé sur une navigation faisant apparaître ou 
disparaître les images (une case, une bulle, l'expression d'un personnage), selon les besoins de la narration. L'expérimentation en classe est réalisée à partir d'un fichier PDF du storyboard où chaque page correspond à une image, permettant une navigation à l'écran proche de celle prévue pour la lecture de la bande dessinée définitive.

La pièce de théâtre choisie pour construire le scénario est Micromégas, le conte de Voltaire dans lequel le philosophe met en scène deux géants (Micromégas et son comparse Saturnien) se déplaçant de planète en planète "grâce aux lois de la gravitation". Ce préalable permet d'envisager le paradoxe suivant: dans les médias, les images d'impesanteur dans lesquelles les astronautes "flottent" suggèrent qu'il n'y a pas de gravitation dans l'espace. Dès lors comment les géants de Voltaire pourraient-ils s'y déplacer "grâce aux lois de la gravitation" ? C'est à cette question que les élèves vont être confrontés, à travers les interrogations des personnages de la bande dessinée.

Conformément aux enseignements de la première expérimentation sur l'épisode $n^{\circ} 1$ (circulation sanguine), l'épisode $n^{\circ} 2$ sur la gravitation comprend 3 chapitres au lieu de 5 pour réduire le temps de lecture. Le problème est par ailleurs plus explicitement présenté dans le récit.

15 L'épisode $\mathrm{n}^{\circ} 2$ intitulé "Bon voyage, Monsieur Newton!" présente les quatre adolescents (les Grandiloquents) dans une salle d'escalade sur bloc où ils tentent d'expérimenter la chute de deux corps sur les tapis d'amortissement. Ils confrontent à cette occasion leurs idées préalables et leurs points de vue sur la notion de gravitation. Plus tard, sur scène, les Grandiloquents mettront à profit leurs réflexions en présentant leur adaptation de Micromégas selon leur interprétation de la physique newtonienne.

\section{Logique du cheminement conceptuel proposé}

L'idée qu'il n'y aurait pas de gravité dans l'espace peut être reliée aux images d'astronautes en impesanteur ${ }^{2}$, très répandues dans les médias. Le terme de gravité étant associé dans le langage courant au phénomène de chute, le fait que les astronautes ne chutent pas mais « flottent » implique a priori une absence de gravité dans l'espace, selon un raisonnement commun qu'on peut résumer de la façon suivante : pas de chute, pas de gravité.

17 L'invalidité de cette logique, du point de vue de la physique, tient au fait que la notion de force de gravité - ou force de gravitation - permet de rendre compte non seulement de la chute, verticale ou parabolique, mais également du mouvement orbital dans l'espace. Or les astronautes en impesanteur dans leur station spatiale, vus du centre de la Terre, ont bien un mouvement circulaire autour de la Terre.

Dépasser la logique initiale "pas de chute, pas de gravité " nécessite de comprendre comment la notion de gravité peut être étendue au mouvement orbital, visuellement très différent de celui de chute. L'enjeu didactique consiste ainsi à mettre en évidence les liens entre la chute sur Terre et le mouvement orbital. Le cheminement conceptuel conçu à cette fin est divisé en deux objectifs intermédiaires.

objectif 1: Mettre en évidence les points communs entre la chute libre et le mouvement orbital à partir de la situation d'impesanteur.

Le premier point commun est que la chute et le mouvement orbital apparaissent tous deux, du point de vue des corps en mouvement, comme des états d'impesanteur. Le second point commun est que ces deux types mouvements ne dépendent pas de la masse. 
Objectif 2 : Justifier les points communs entre chute et mouvement orbital par leur continuité, en considérant la vitesse par rapport au sol.

La continuité entre les deux mouvements peut être visualisée avec l'expérience de pensée du "canon de Newton", où sont considérées les différentes trajectoires de chute d'un objet selon la vitesse fournie au départ. La chute verticale, la chute parabolique et le mouvement orbital peuvent alors être vus comme trois cas d'un même type de mouvement, à savoir le mouvement lié à la gravitation, justifiant ainsi les points communs mis en évidence.

Le cheminement conceptuel permettant d'atteindre ces objectifs didactiques est explicité directement dans le contexte du récit proposé, en relation avec les choix scénaristiques et graphiques.

\section{Articulation des choix didactiques, scénaristiques et graphiques}

\section{Contexte et position du problème}

21 Le contexte de la mise en scène choisi est celui d'une salle d'escalade sur bloc, où les personnages peuvent expérimenter par eux-mêmes la chute libre sur des tapis d'amortissement.

\section{Exposer la situation et faire émerger un prérequis}

Une scène d'introduction (Annexes 1) permet de rappeler une donnée nécessaire à la compréhension du récit : le fait que la chute ne dépende pas de la masse. Alors que deux personnages de masse visiblement différente tombent simultanément sur les tapis d'amortissement, ceux-ci évoquent l'expérience de Galilée selon laquelle la vitesse de chute des objets ne dépend pas de leur masse, lorsque l'influence de l'air peut être négligée (figure 1). S'appuyant sur une analogie possible entre une expérience historique et une expérience actuelle, la bande dessinée interpelle ici les élèves sur un supposé prérequis.

Figure 1 : écrans 15,16 et 17 du storyboard, épisode $n^{\circ} 2$ sur la gravitation 


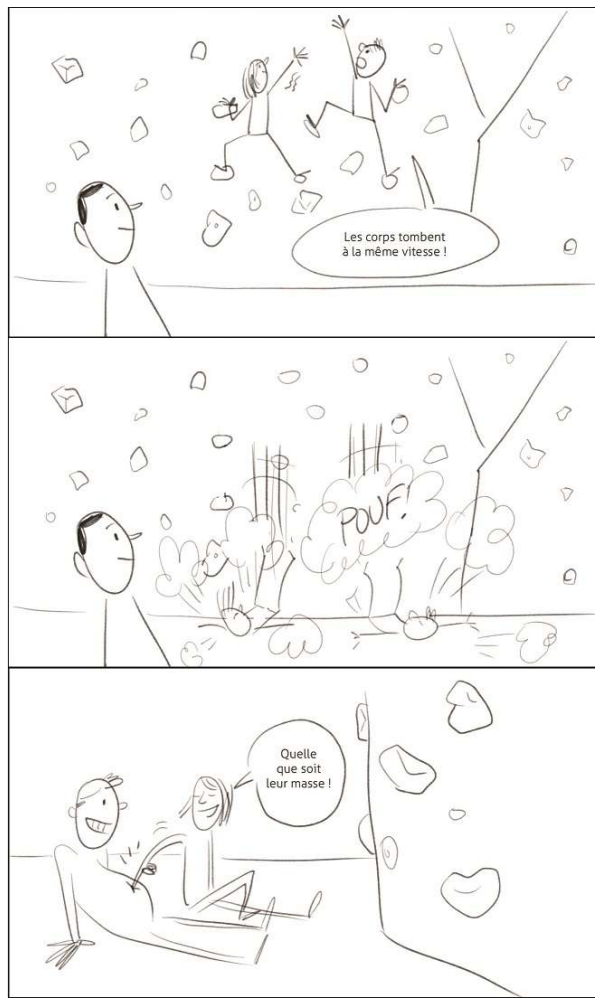

Govin et Bordenave, « tous droits réservés », 2019

\section{Déclencher le récit et positionner le problème}

D'après les images d'astronautes en impesanteur, il n'y pas de "gravité" dans l'espace, au sens de la chute des objets. Mais alors comment serait-il possible de se déplacer "grâce" à la gravitation, tel que le suggère le texte de Voltaire ? Ce hiatus est expressément mis en scène dans l'épisode (Annexes 2) par un dialogue entre les personnages interpellés par la tirade d'Iris à propos de Micromégas censé “ [aller] de globe en globe, lui et les siens...Comme un oiseau voltige de branche en branche. ". Les protagonistes basculent dans la résolution d'un problème tandis que les élèves, en tant que lecteurs du récit, sont invités à suivre cette résolution par l'effet déclencheur de la séquence narrative (figure 2). 
Figure 2 : écran 25 du storyboard, épisode $n^{\circ} 2$ sur la gravitation

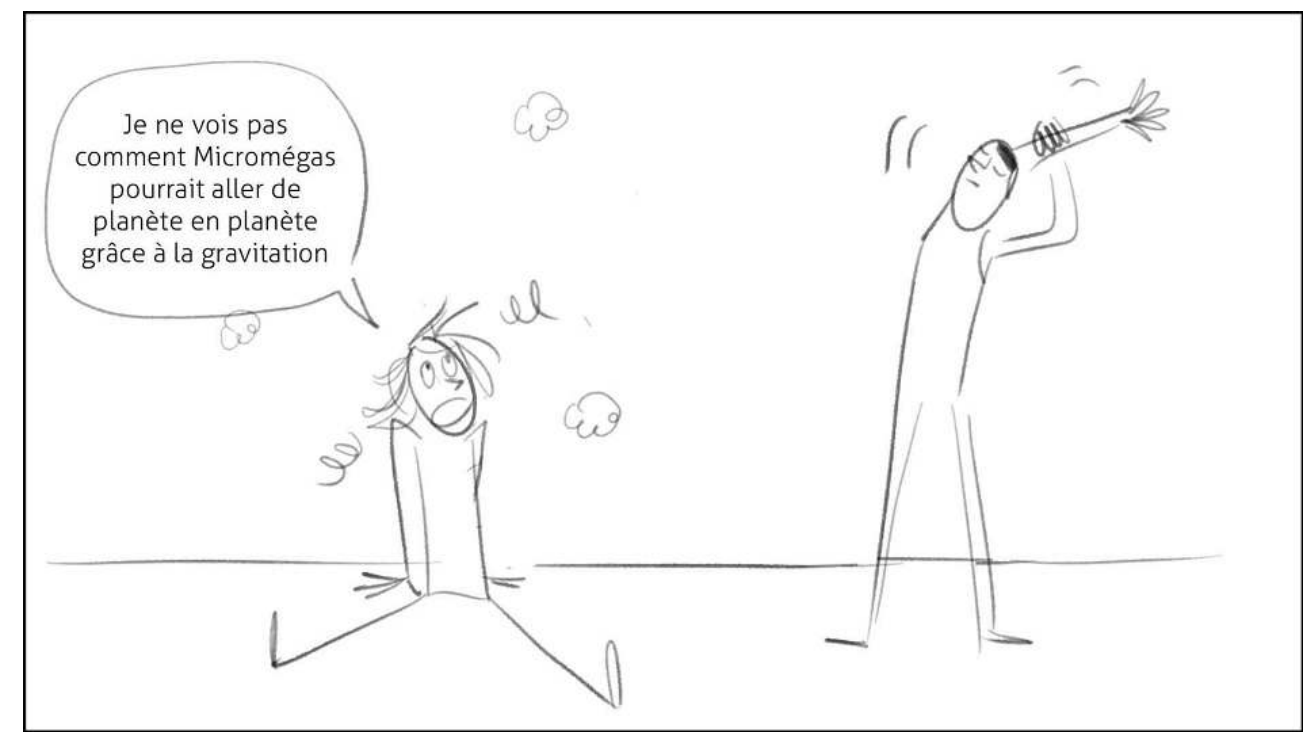

Govin et Bordenave, «tous droits réservés », 2019

\section{Convoquer des images de pensée}

Le flottement des astronautes est évoqué par la voix de Tom et par l'image représentant Iris en astronaute dans une station spatiale, en difficulté avec du dentifrice à poser sur sa brosse à dent (figure 3). Cette image de pensée, cocasse et irréaliste, est la première intrusion dans le récit jusque-là réaliste d'une représentation imaginaire. Sa place dans le récit graphique, possible grâce aux propriétés du genre fictionnel, permet de clarifier l'image mentale référentielle - tout le monde connaît les images médiatiques d'astronautes dans une station spatiale - tout en rythmant la lecture par des touches humoristiques dans le récit. 
Figure 3 : écrans 26 à 28 du storyboard, épisode $n^{\circ} 2$ sur la gravitation

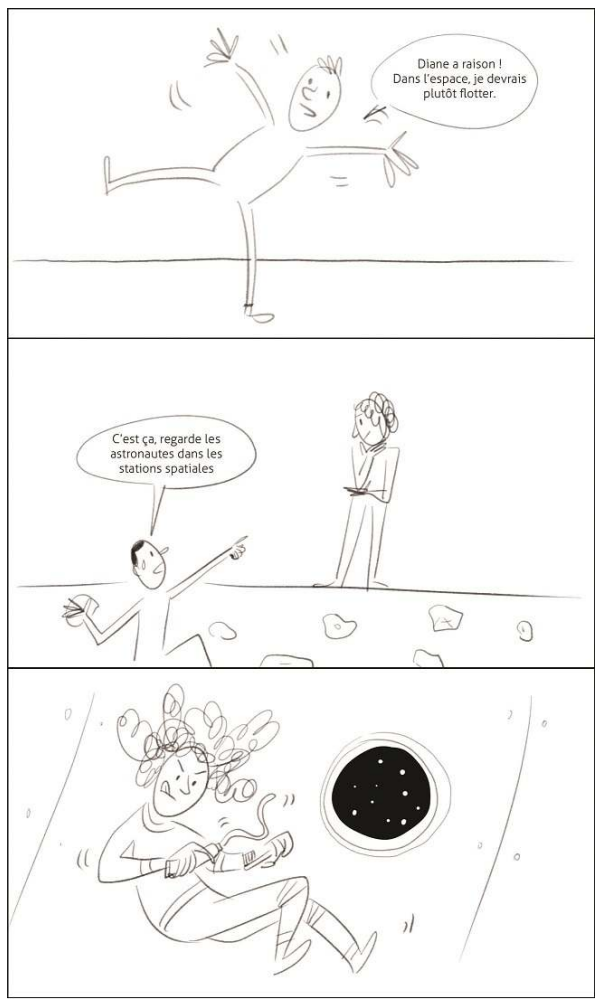

Govin et Bordenave, « tous droits réservés », 2019

\section{Objectif didactique $n^{\circ} 1$ : points communs entre chute et mouvement orbital}

\section{Une expérience de pensée mise en scène grâce à la bande dessinée numérique}

La suite du cheminement vise à mettre en évidence un premier point commun entre chute et mouvement orbital : leur lien à l'état d'impesanteur, en changeant de point de vue. La première étape consiste à considérer le cas de la chute libre du point de vue d'une personne qui chute, via une expérience de pensée. Le contexte de la salle d'escalade permet de mettre en scène cette expérience : Tom et Titouan se laissent tomber d'un mur d'escalade sur un tapis (Annexes 3). Puisque leur vitesse ne dépend pas de leur masse, ils tombent à la même vitesse et se voient ainsi "flotter" l'un par rapport à l'autre.

Deux spécificités du média bande dessinée sont exploitées dans cette mise en scène. D'une part, le ralentissement du temps est visualisé par séquençage de la chute en plusieurs actions qui, dans la réalité, ne seraient pas perceptibles. D'autre part, le "pacte fictionnel", grâce auquel le lecteur feint de penser que ce que [les auteurs] nous raconte [nt] est réellement arrivé (Eco, 1996), invite à accepter le caractère irréaliste de ce ralentissement, compris implicitement comme visant à attirer l'attention sur cet instant. Ce choix de mise en scène graphique est prévu pour être enrichi par l'outil numérique dans la bande dessinée finalisée : la séquence visuelle sera animée par le défilement de l'arrière-plan au moyen d'un GIF animé, tandis que les personnages qui chutent resteront statiques à l'écran. Il est ainsi explicitement montré que des corps en mouvement (chute libre) peuvent paraître en impesanteur de leur propre point de vue. 


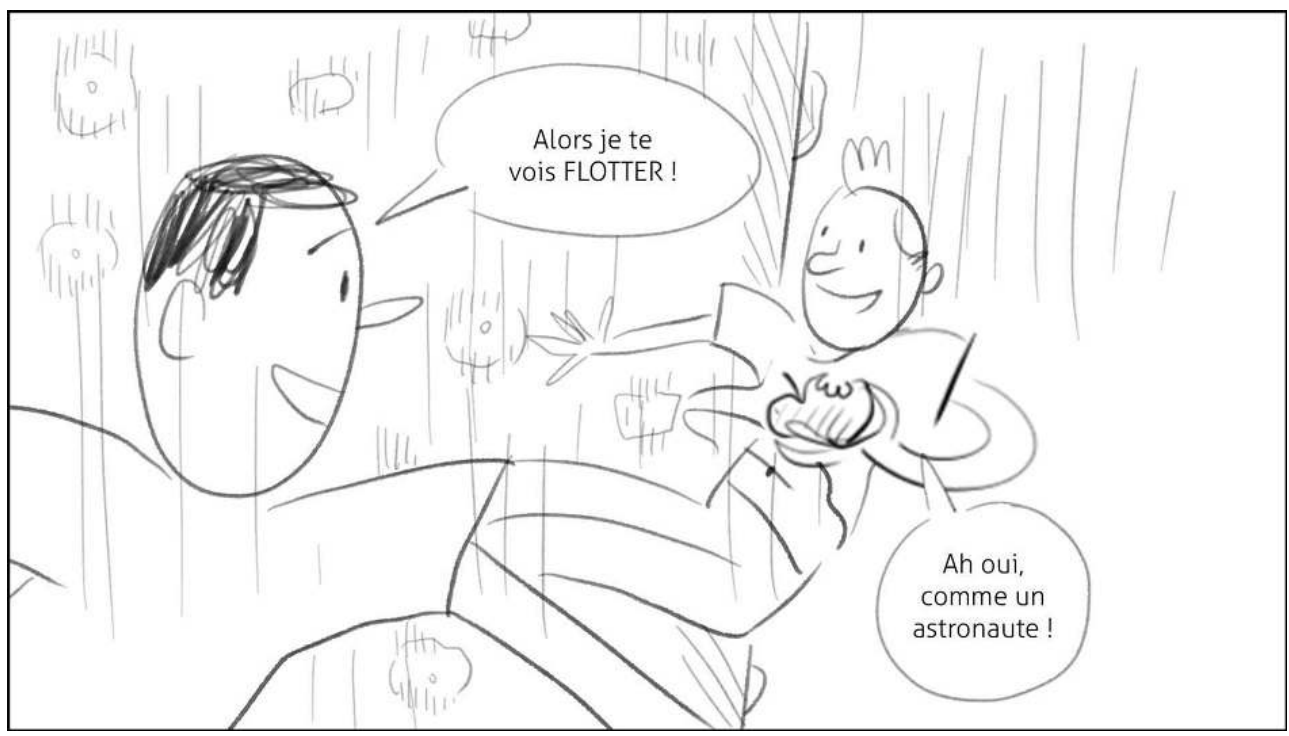

Govin et Bordenave, « tous droits réservés », 2019

\section{Le séquençage des images comme outil de questionnement}

La conception de la scène de la chute simultanée (figure 5) a fait l'objet d'une coconstruction progressive combinant les intentions didactiques et narratives. Dans une première version du scénario, Tom ne tenait pas de pomme dans sa main. Mise en image dans le storyboard, cette situation mettait en scène une différence de masse entre les deux adolescents trop équivoque. L'ajout d'un petit objet que l'un d'eux lâche au cours de la chute a permis de signifier plus explicitement que le mouvement de chute est identique quelle que soit la masse des objets. Le choix d'une pomme pour représenter cet objet permet de combiner l'enjeu didactique (visualiser la chute de corps de masse suffisamment différente) et la cohérence narrative (en clin d'oeil à la mythique pomme de Newton). Envisagé pour une navigation sur écran, le séquençage des images peut alors permettre de valoriser ce changement pour qu'il suscite le questionnement suivant : au moment où Tom desserre les doigts, qu'arrive-t-il à la pomme ? Va-t-elle tomber plus vite, moins vite ou de la même façon que les personnages? Lors d'une lecture collective en classe par vidéo-projection dont le rythme est contrôlé par l'enseignant, ce séquençage permet l'exploitation de ces questionnements. La réponse suggérée par le découpage en 3 étapes (figure 5) permet de mettre en évidence que la vitesse de la pomme est la même que celle de Tom et Titouan, puisqu'elle "flotte" relativement à eux. La mise en scène de cette expérience de pensée propre au récit fictionnel nous permet "de nous inscrire dans un processus de questionnement à propos du réel, mais aussi dans un travail de projection de nos propres représentations" (Triquet \& Bruguière, 2014). 
Figure 5 : écrans $41-43$ du storyboard, épisode $n^{\circ} 2$ sur la gravitation

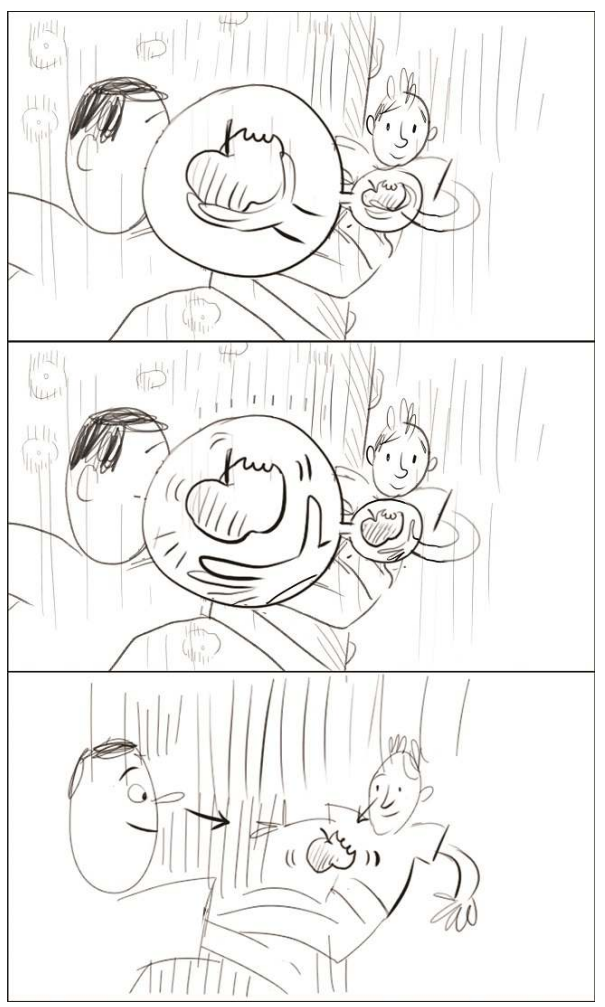

Govin et Bordenave, « tous droits réservés », 2019

Un pacte fictionnel en BD pour interroger les élèves et favoriser les analogies depuis le sol (Annexes 4). La compréhension que l'état d'impesanteur dépend du point de vue est extrapolée aux astronautes. En se demandant quel pourrait être leur mouvement d'un autre point de vue, les personnages prennent conscience que l'astronaute et sa station spatiale tournent autour de la Terre. Il s'agit d'un premier élément face au problème, qui consistait à se demander comment Micromégas pourrait se déplacer dans l'espace, alors que les astronautes, eux, flottent.

La dernière étape est la compréhension que le mouvement orbital ne dépend pas de la masse : l'astronaute et la station spatiale ont le même mouvement malgré leur masse différente, de la même façon que pour la chute. Cela permet à l'un des personnages de faire le lien avec la gravité sur Terre, et par extrapolation, d'apporter une justification au propos initial de Voltaire: Micromégas pourrait donc effectivement se déplacer dans l'espace grâce à la gravitation.

Cette scène implique une mise en situation hybride mêlant une image mentale d'un personnage (Iris) en orbite dans l'espace avec l'apparition d'une image "réaliste" de personnages sur Terre (Tom et Diane) dont la lecture permet de transposer le point de vue du lecteur dans l'espace (figure 6). Le médium bande dessinée permet également de juxtaposer les deux environnements (terrestre et spatial) en une image unique sans que la lecture du récit ne soit invraisemblable. Ici, les auteurs font le pari de la fiction comme contexte narratif permettant la vraisemblance de la situation. 
Figure 6 : écrans 48 et 51 du storyboard, épisode $n^{\circ} 2$ sur la gravitation

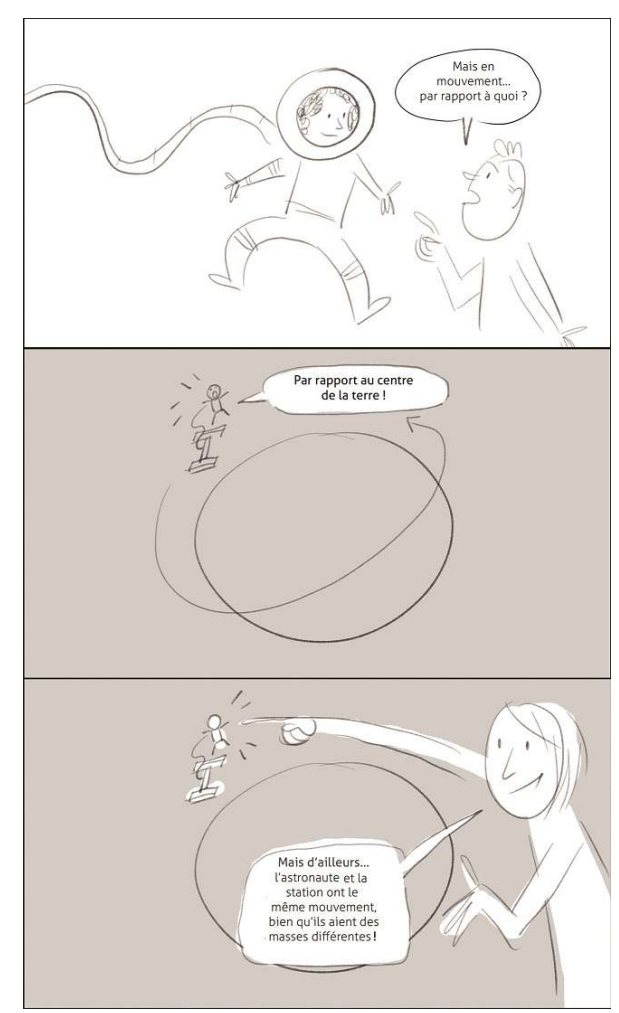

Govin et Bordenave, « tous droits réservés », 2019

\section{Objectif didactique $n^{\circ} 2$ : continuité entre chute et mouvement orbital}

Le second objectif didactique consiste à mettre en évidence la continuité entre le mouvement de chute et le mouvement orbital, en s'appuyant sur le schéma du " canon de Newton » (figure 7), proposée par Newton dans "De mundi Systemate" en 1685 (publié en 1728). Cette expérience de pensée représente différentes trajectoires de chute d'un boulet de canon selon la vitesse fournie. Elle permet d'imaginer comment la chute parabolique, en augmentant la vitesse initiale, peut devenir une chute allant si loin que le rapprochement du sol soit compensé par la courbure de la Terre. L'objet se rapproche d'autant que la Terre "s'éloigne" en se courbant, au point qu'il fasse le tour: c'est le mouvement orbital autour de la Terre. 


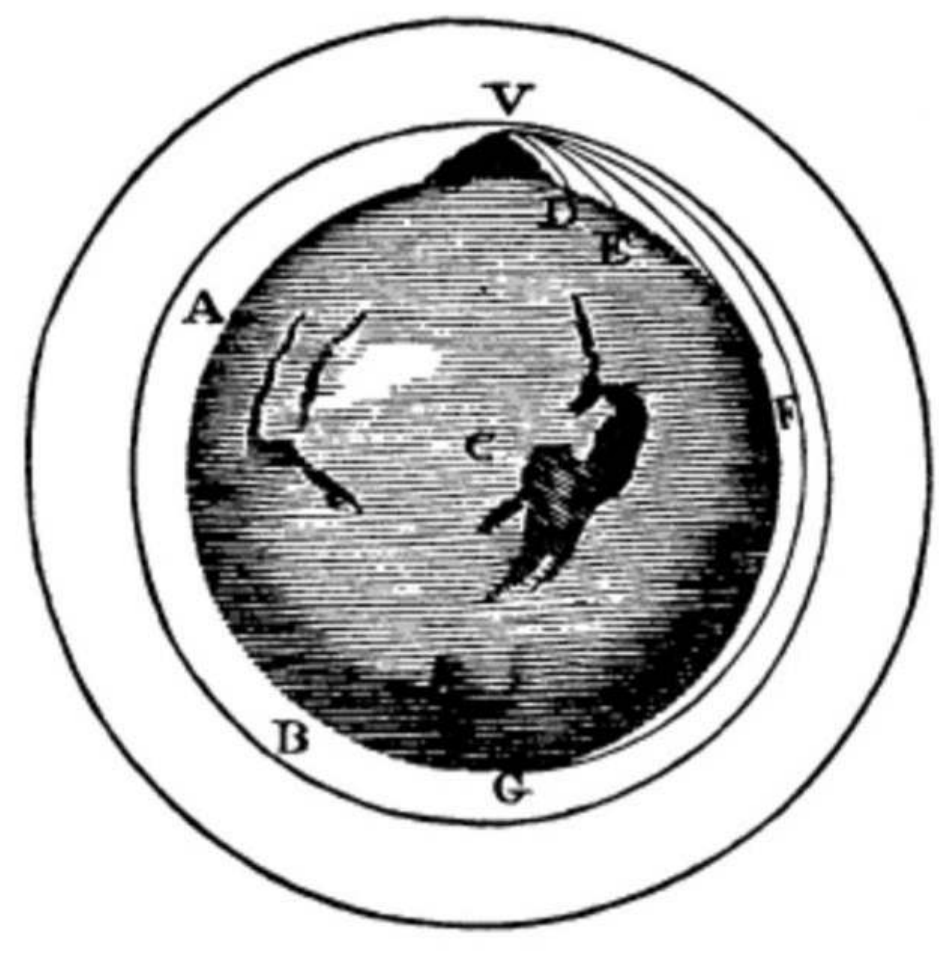

La chute verticale, la chute parabolique et le mouvement circulaire autour de la Terre pourraient donc être vus comme trois cas d'un même type de mouvement : le mouvement gravitationnel. Cette idée permet de comprendre les points communs mis en évidence entre la chute et du mouvement orbital (objectif 1), dont l'indépendance de la masse.

\section{Une fiction théâtrale dans la fiction dessinée : vertu des récits emboités}

$33 \mathrm{Au}$ chapitre 3 de l'épisode, les Grandiloquents, en répétition sur la scène d'un théâtre, jouent l'adaptation du final de Micromégas qu'ils ont imaginée. Appuyant son propos par un schéma (le "canon de Newton") dont il est l'auteur, Titouan (déguisé en Newton) demande à Tom et Diane (déguisés en géants) de l'aider à faire une expérience pour vérifier l'hypothèse selon laquelle un objet lancé assez fort depuis le sommet d'une montagne ne retomberait pas sur Terre (figure 8). 
Figure 8 : écran 72 du storyboard, épisode $n^{\circ} 2$ sur la gravitation

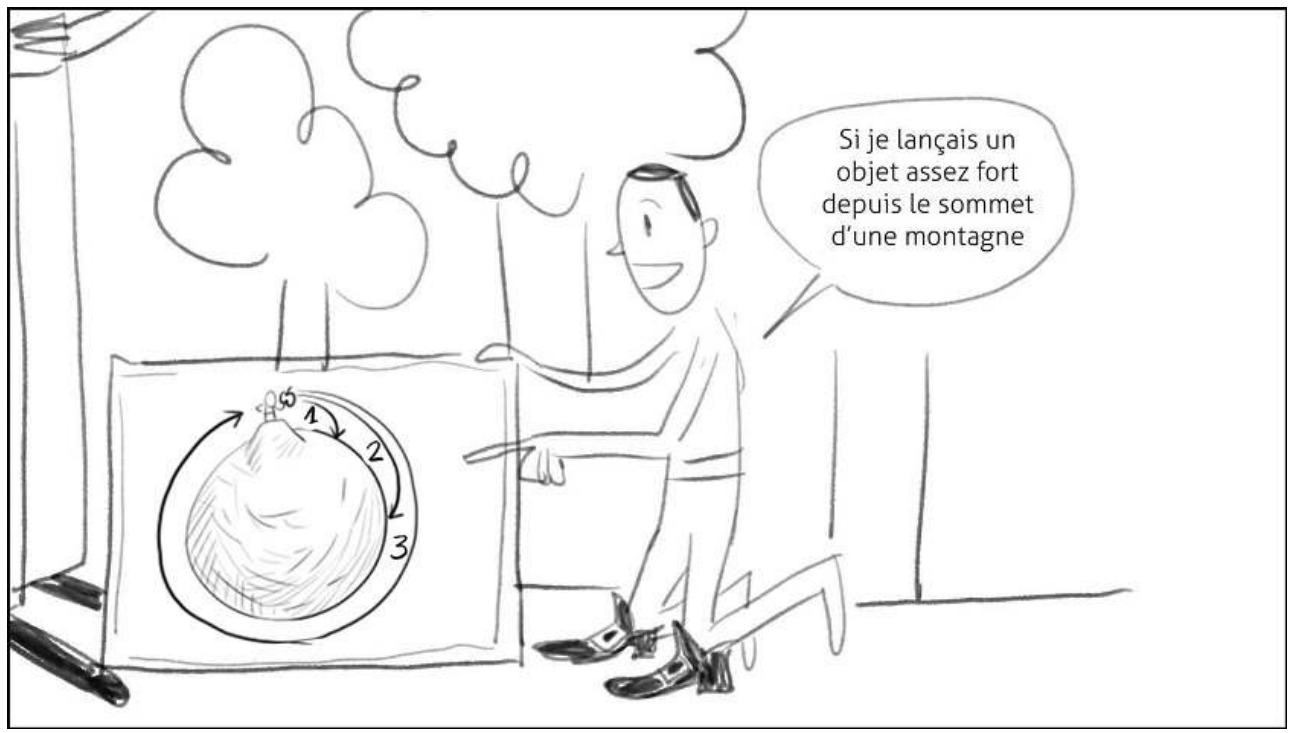

Govin et Bordenave, « tous droits réservés », 2019

Dans ce chapitre (Annexes 5), un second niveau de fiction vient se superposer au premier. Parce qu'ils sont sur scène et qu'ils jouent la comédie, les Grandiloquents deviennent les protagonistes d'un second récit fictionnel : la pièce de théâtre qu'ils ont adaptée du conte de Voltaire. Avec ce dispositif de narration, un degré de liberté supplémentaire est donné au discours prononcé par les jeunes comédiens. Dès lors, quand Newton (joué par Titouan) prend la parole, le personnage présent à l'écran n'est pas la représentation fidèle du célèbre savant et son discours n'est qu'une interprétation possible du discours scientifique, technique scénaristique évitant de trahir les discours d'une personnalité historique.

Par ailleurs, la formulation de l'hypothèse énoncée par Titouan (Newton) est interprétée à l'écran par une image de pensée dans l'esprit des deux géants (Tom et Diane), moyen propre à la bande dessinée pour donner à voir, sans les expliciter, les références aux expériences discutées en amont dans le récit.

Enfin, l'expérience newtonienne est ainsi représentée (Annexes 6) : Tom (Micromégas) jette alors dans les airs un Playmobil figurant le petit homme qu'est Newton comparé aux géants (figure 9). Lancé d'un côté de la scène, le playmobil-Newton resurgit depuis l'autre côté, mimant ainsi un invraisemblable tour de la Terre. Images enchaînées sans commentaire, la scène théâtrale qui clôture également l'épisode de bande dessinée est laissée à l'interprétation des élèves. C'est une fin ouverte susceptible d'ouvrir un débat en classe sur l'expérience proprement dite. 
Figure 9 : écran 82 du storyboard, épisode $n^{\circ} 2$ sur la gravitation

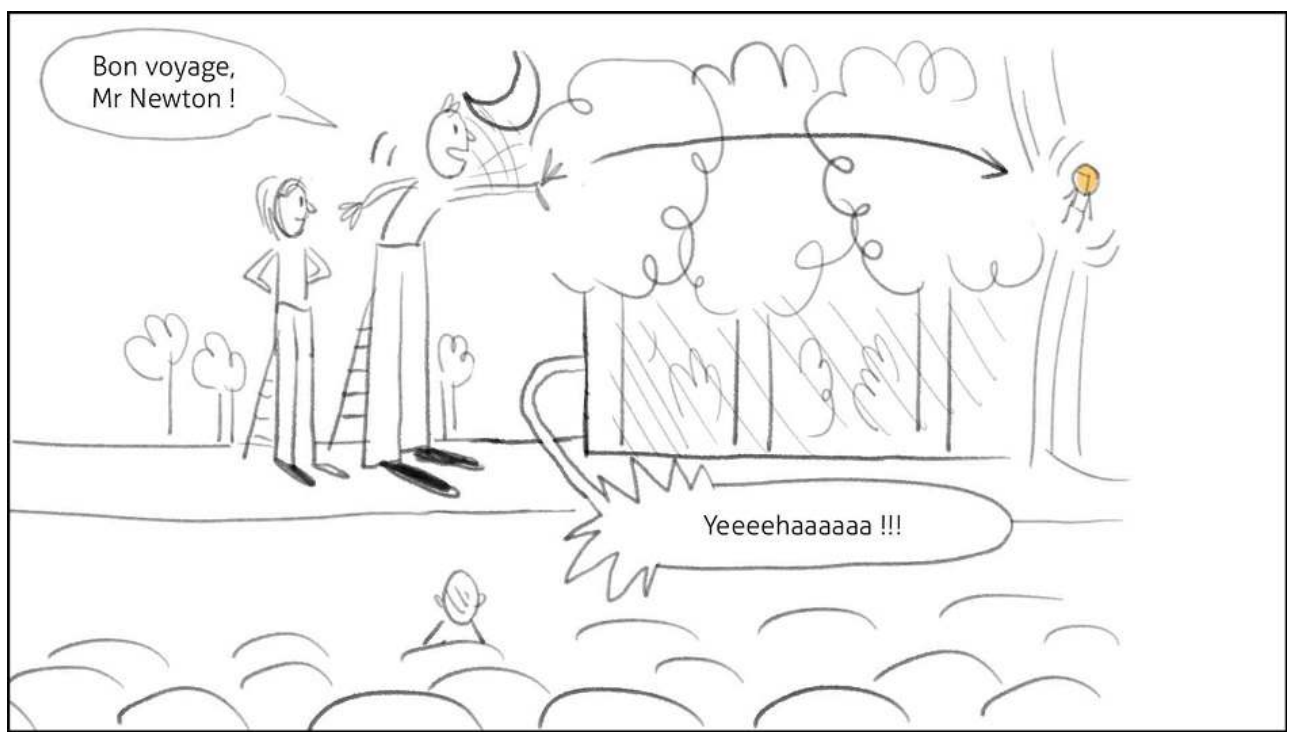

Govin et Bordenave, « tous droits réservés », 2019

\section{Expérimentation du storyboard en classe}

\section{Méthodologie}

Le storyboard de l'épisode sur la gravitation a été expérimenté avec une classe de seconde générale et technologique dans laquelle enseigne un des auteurs. Bien que de niveau supérieur au niveau ciblé par la ressource (cycle 4), les élèves ont été choisis pour participer de manière plus efficace au processus de conception de la série: les auteurs comptent en effet sur une plus grande capacité chez ces lecteurs à exprimer leurs erreurs ou leur incompréhension.

L'expérimentation s'est déroulée au cours d'une séance de $1 \mathrm{~h} 30$, en commençant par une lecture du storyboard sur écran d'ordinateur, suivie par une activité en binôme sur les différentes scènes clefs et le renseignement d'un questionnaire. Vingt-et-un élèves étaient présents, soit 10 binômes et un trinôme.

Cette première expérimentation exploratoire vise à recueillir des informations permettant d'orienter la conception d'une version améliorée du storyboard avant la réalisation de la bande dessinée proprement dite, ainsi que la conception de ressources d'accompagnement destinées aux enseignants impliqués dans l'utilisation de l'épisode. Les données obtenues permettront également de guider la mise en place d'une nouvelle expérimentation en classe, en précisant les questions de recherche et la méthodologie.

Les différentes phases de l'expérimentation, les questions de recherche associées, et le type de données recueillies sont synthétisées dans le tableau ci-dessous :

Tableau 1 : Protocole expérimental de l'utilisation en classe

\begin{tabular}{|l|l|l|l|}
\hline $\begin{array}{l}\text { Questions } \\
\text { recherche }\end{array}$ & de & $\begin{array}{l}\text { Exemples de questions posées } \\
\text { aux élèves }\end{array}$ & Données recueillies \\
\hline
\end{tabular}




\begin{tabular}{|c|c|c|c|}
\hline 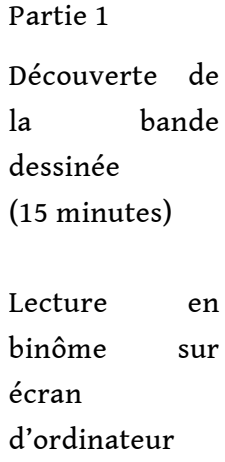 & $\begin{array}{l}\text { Quel est l'impact de } \\
\text { la première lecture } \\
\text { chez les élèves ? } \\
\text { (évaluation de } \\
\text { l'appréciation et de } \\
\text { la clarté du récit) }\end{array}$ & $\begin{array}{l}\text { "Indiquer les numéros des } \\
\text { pages }^{3} \text { où ce n'est pas clair } \\
\text { pour vous " et celles « qui } \\
\text { vous ont particulièrement plu } \\
\text { " } \\
\text { Demande d'explicitation à } \\
\text { l'écrit. }\end{array}$ & $\begin{array}{l}\text { Numéros de pages et } \\
\text { traces écrites des } \\
\text { justifications }\end{array}$ \\
\hline $\begin{array}{l}\text { Partie } 2 \\
\text { Relecture } \\
\text { détaillée } \\
\text { (40 minutes) } \\
\text { Relecture des } \\
\text { scènes clefs } \\
\text { guidées par une } \\
\text { question et } \\
\text { discussion en } \\
\text { binôme }\end{array}$ & $\begin{array}{lrr}\text { Quelle } & \text { est } & \text { la } \\
\text { compréhension } & \text { des } \\
\text { élèves des } & \text { scènes } \\
\text { clefs, } & & \text { sans } \\
\text { intervention } & \text { de } \\
\text { l'enseignant? } & \\
\text { Dans quelle } & \text { mesure } \\
\text { les élèves } & \text { sont-ils } \\
\text { capables } & \text { de } \\
\text { mobiliser ce } & \text { qu'ils } \\
\text { ont compris de la BD } \\
\text { dans des situations } \\
\text { différentes? }\end{array}$ & $\begin{array}{l}\text { "Relire les pages } 33 \text { à } 53 \text { et } \\
\text { discuter en binôme de ce que } \\
\text { vous avez compris de cette } \\
\text { partie de la BD. Après cette } \\
\text { discussion, expliquez avec vos } \\
\text { propres mots les liens entre } \\
\text { l'expérience proposée par } \\
\text { Titouan (p.33) et la situation } \\
\text { d'impesanteur dans l'espace } \\
\text { (p.47).» }\end{array}$ & $\begin{array}{l}\text { Enregistrements audio } \\
\text { des discussions en } \\
\text { binôme et capture } \\
\text { vidéo des écrans } \\
\text { d'ordinateur }\end{array}$ \\
\hline $\begin{array}{l}\text { Partie } 3 \\
\text { Perception et } \\
\text { appréciation } \\
\text { globale de la } \\
\text { bande dessinée } \\
\text { (15 minutes) }\end{array}$ & $\begin{array}{l}\text { A l'issue du travail, } \\
\text { quelle perception } \\
\text { des élèves de la } \\
\text { difficulté, de l'utilité, } \\
\text { et du caractère } \\
\text { motivant de la bande } \\
\text { dessinée? }\end{array}$ & $\begin{array}{l}\text { «Cette activité vous a-t-elle } \\
\text { permis de comprendre } \\
\text { quelque chose de nouveau } \\
\text { concernant la gravitation?" } \\
\text { " Le format BD vous a-t-il } \\
\text { motivé pour essayer de } \\
\text { comprendre un sujet } \\
\text { scientifique?» }\end{array}$ & $\begin{array}{l}\text { Questionnaires à choix } \\
\text { multiples et réponses } \\
\text { aux } \\
\text { ouvertes. }\end{array}$ \\
\hline
\end{tabular}

\section{Résultats \& discussions}

Partie 1 - Découverte de la $\mathrm{BD}$ : quel impact de la première lecture ?

\section{Peu de difficultés de lecture}

41 La première lecture de la bande dessinée semble fluide pour la majorité des élèves. A la question des "pages qui ne sont pas claires pour vous", 9 élèves sur 21 n'en mentionnent aucune. Parmi les pages indiquées comme pas claires, aucun consensus particulier n'émerge. Seule une page est mentionnée par 2 élèves : celle où apparaît la distinction entre les termes de gravité et de gravitation. Cette difficulté invite à réfléchir sur le moment d'aborder cette distinction de vocabulaire dans le travail avec la bande dessinée. 


\section{L'humour est plébiscité}

Concernant les "pages qui vous ont particulièrement plus", quatre passages sont cités entre 6 et 15 fois. Ceux-ci ont pour point commun la chute des personnages, dont la mise en image a interpellé les élèves :

- les écrans 15 à 17 : chute commune de Diane et Tom, pour 6/21 élèves.

- les écrans 21 à 24 : chute de Diane, pour 6/21 élèves.

- les écrans 33 à 44 : chute simultanée de Tom, Titouan et la pomme pour 15/21 élèves.

- les écrans 78 à 84 : expérience finale du "lancé de Newton" sur la scène du théâtre, pour 8/21 élèves.

Les élèves apprécient positivement l'humour dans les scènes préférées (13/15 commentaires). D’autres commentaires (4) évoquent la clarté des séquences : "Les scènes sont claires et drôles", "La façon dont le mouvement d'un objet par rapport à un autre est amusante et compréhensible", "On comprend toute la bande dessinée", ou encore "Cela m'a fait apprendre des choses".

4 Dans quatre autres commentaires, la réalisation d'expériences par les personnages semble marquer positivement les élèves, par exemple : "J'ai aimé ces pages car ils ont testé pour voir si ils tombent à la même vitesse", "Ces quelques pages m'ont plu car c'est le moment où il mettent en exécution leur expérience", "L'expérience n'est pas totalement possible donc c'est marrant".

\section{Une difficulté inattendue : l'indépendance de la masse lors de la chute}

Les enregistrements des discussions au sein de trois binômes révèlent que l'indépendance de la masse lors de la chute est une information nouvelle pour certains élèves, et non un prérequis comme supposé par les auteurs. Un élève s'étonne par exemple : "Ils tombent à la même vitesse, quelle que soit leur masse, je savais pas ça, ça m'intéresse". Le caractère contre intuitif de cette proposition est soulevé un autre élève : "Monsieur ça c'est faux. Non enfin c'est pas faux mais c'est étonnant parce nous quand on fait tomber deux objets qui ont pas du tout le même poids ils tombent pas en même temps". Deux binômes font une expérience de chute simultanée de deux objets de masse différente, sans réussir à conclure. Bien que déjà vu en classe au cours de l'année, l'indépendance de la masse lors de la chute reste un sujet de discussion entre les élèves.

Nous pouvons supposer que l'effort fourni par les élèves pour accepter l'indépendance de la masse rend d'autant plus difficile leur appropriation de la suite du raisonnement. Cette observation est cruciale pour l'usage de l'épisode en classe : le traitement de la chute libre en amont de la lecture est indispensable afin que l'indépendance de la masse lors de la chute puisse être considérée comme un rappel.

Partie 2 - Relecture détaillée : quelle compréhension des scènes clefs ?

\section{Objectif didactique $n^{\circ} 1$ : liens entre la chute et l'impesanteur}

La première question posée concerne la compréhension de la scène de chute simultanée de Titouan, Tom et la pomme, et ses liens possibles avec un astronaute en orbite autour 
de la Terre (voir énoncé dans le Tableau 1, Partie 2). Deux liens sont attendus entre la chute et la situation d'impesanteur :

- l'état d'impesanteur ou de mouvement dépend du point de vue,

- la chute et le mouvement orbital ne dépendent pas de la masse.

D'après les enregistrements et les traces écrites de l'activité, seuls 2 élèves sur 21 expriment ces deux liens par eux-mêmes, sans intervention de l'enseignant. Trois élèves saisissent seulement le premier lien, et 2 saisissent seulement le second.

Cette incompréhension très majoritaire de la scène centrale peut être rapprochée dans un premier temps du sentiment de clarté et d'amusement exprimé par la grande majorité des élèves. La plupart d'entre eux ne comprennent pas la logique du raisonnement présenté, sans pour autant ressentir de difficulté ou de confusion lors de la lecture. Leur intérêt pour la narration et la mise en image amusante de ce passage semble faire écran à la difficulté conceptuelle que la scène supporte. Une question de recherche pour une future expérimentation serait dès lors de déterminer l'impact de l'humour dans la lecture du récit vis-à-vis de la compréhension du raisonnement sous-jacent. Il s'agirait de déterminer si la lecture de scènes humoristiques portant une information didactique clef, est un paramètre favorisant ou au contraire faisant obstacle à la perception de l'information.

D'autre part, le faible taux de compréhension obtenu lors de la première question indique que celle-ci nécessite d'être explicitée davantage, afin de guider plus progressivement les élèves vers les mises en relation recherchées. Une série de questions intermédiaires, pour une future expérimentation, pourraient consister à questionner d'une part les deux mouvements relativement à chacun des points de vue (interne ou lié à la Terre), puis demander de comparer explicitement les masses dans les deux cas (voir annexe 7A pour une formulation explicite de ces questions).

\section{Objectif didactique $n^{\circ} 2$ : continuité entre chute et mouvement orbital}

51 La deuxième question posée dans l'activité concerne la comparaison de plusieurs trajectoires montrant la continuité entre la chute courbe et le mouvement orbital (voir Figure 6 : écran 72 du storyboard) : "Quels sont les points communs ou différences entre les trajectoires 1,2 et 3 concernant:

- le rôle de la masse (le mouvement serait-il le même si la masse de l'objet était différente?),

- la valeur de la vitesse transmise à l'objet au moment du lancer ?"

Le point commun attendu est que pour chaque cas le mouvement ne dépend pas de la masse, et la différence attendue est la vitesse de lancer pour chaque cas.

52 A cette question, 7 élèves indiquent que le mouvement serait le même si la masse était différente, et 8 (dont 6 des précédents) indiquent que le mouvement dépend de la vitesse. Pour les élèves répondant correctement, la capture de la navigation sur écran permet de constater des allers-retours dans le récit pour repérer les différentes scènes de la bande dessinée utiles pour leurs réponses. C'est le cas par exemple d'un élève revenant sur la scène de la chute de Diane et Tom (écrans 16-17) pour argumenter :

“- En gros est-ce que si la masse de l'objet est différente est-ce que il tombera de la même façon? Plus vite, moins vite?

- Ben il avait pas dit que tout tombe à la même vitesse je sais pas quoi là.

- que ils tombaient en même temps. Ouais ben attend regarde, tu vas voir [retour à 
la p. 16-17] quand ils vont tomber ils vont tomber en même temps j'crois. Ben oui parce que...

- ...les corps tombent à la même vitesse, quelle que soit leur masse !

- Ben moi je dis la masse n'a pas d'impact."

53 Cet exemple montre que les élèves ont pu s'appuyer sur des informations qu'ils ont localisées dans le récit, et s'y référer pour argumenter leur propos dans le débat. Cette observation illustre un des intérêts du médium bande dessinée dont les séquences visuelles peuvent s'avérer propices à la mémorisation de situations-clefs. La lecture sur écran quant à elle, peut favoriser les allers-retours dans le récit rapidement.

La faible proportion d'élèves réussissant à répondre correctement montre de nouveau que la question n'est pas suffisamment guidée pour être accessible à une majorité d'élèves. Une première suggestion serait l'ajout du cas de la chute verticale sur le schéma de Newton (figure 6) de façon à faciliter le lien avec la question précédente. Avec cette modification, la question pourrait alors être reformulée en proposant d'imaginer le point de vue des personnages en chute s'ils étaient lâchés ou lancés plus ou moins fort par le géant, selon chacune des trajectoires du schéma (voir annexe 7B pour une formulation complète des questions). Considérer ces différents cas permet de comprendre le lien entre l'état d'impesanteur lors la chute verticale et l'état d'impesanteur en orbite : quelle que soit la vitesse de lancer, les personnages et la pomme ayant la même vitesse initiale, ils ont tous le même mouvement, et donc de leur point de vue, flottent les uns par rapport aux autres.

\section{Partie 3 - Perception et appréciation globale de la bande dessinée}

La dernière partie de l'expérimentation consiste en un court questionnaire sur la perception générale de la bande dessinée.

\section{Appréciation plutôt positive de la bande dessinée}

Environ la moitié des élèves a apprécié positivement la lecture du storyboard parce qu'ils l'ont jugé amusant (12/21) et/ou instructif $(8 / 21)^{4}$. A la question « Le format BD vous a-t-il motivé pour essayer de comprendre un sujet scientifique ? ${ }^{5}$ ", la moitié des élèves a trouvé que le format BD a répondu positivement (5 "oui", 6 "oui beaucoup") et 2 "pas vraiment".

L'identification aux personnages est variable, avec une prédominance pour Titouan, en raison de l'expérience de saut qu'il suggère et qu'il met en œuvre ${ }^{6}$. Quatre élèves ne se sont identifiés à aucun des personnages.

\section{Perception de la difficulté de l'activité}

Trois élèves indiquent avoir perçu l'activité comme "facile" ou "très facile" majorité des élèves (17/21) comme "ni trop facile, ni trop difficile". Aucun élève n'a perçu l'activité comme "difficile" ou "très difficile, malgré la faible proportion ayant réussi à comprendre le cheminement conceptuel proposé. Ce constat nous incite à nouveau à questionner le lien entre l'appréciation du récit - en particulier sa tonalité humoristique et la compréhension effective du raisonnement qu'il sous-tend. Ce point devra être questionné lors de la prochaine expérimentation. Par ailleurs, 9/21 élèves déclarent que l'activité sur la BD leur a permis de comprendre quelque chose de nouveau sur la 
gravitation. Cependant cinq parmi eux mentionnent l'indépendance de la masse lors de la chute. Cela montre à quel point cet aspect, considéré a priori comme un rappel d'un fait connu, les a marqués au point d'être le principal point retenu.

\section{Conclusion}

Notre travail a consisté à concevoir et à expérimenter un support d'enseignement en sciences physiques sur la gravitation sous la forme d'un épisode de bande dessinée numérique intitulé «Bon voyage, Monsieur Newton!». Inclus dans la série «Les Grandiloquents » en cours de développement, l'épisode sur la gravitation a fait l'objet d'une co-construction associant scénariste, dessinatrice et chercheur en didactique de la discipline. Cette co-écriture permet d'élaborer un récit au sein duquel les intentions didactiques contraignent ou se nourrissent du format bande dessinée. Contrairement aux bandes dessinées scientifiques habituelles, la série n'a pas pour objectif de fournir des informations scientifiques au lectorat mais plutôt de questionner les élèves et de les guider dans le suivi d'un raisonnement, grâce à l'accompagnement de leur professeur.

La conception en question dans l'épisode est l'idée selon laquelle il n'y a pas de gravité dans l'espace. La première étape de la stratégie didactique proposée consiste à mettre en évidence, à partir de l'état d'impesanteur, que le mouvement d'astronautes en orbite ne dépend pas de la masse, de même que pour la chute libre. Cette proposition permet de relier la gravité terrestre et le mouvement orbital en amont du concept plus général de force gravitationnelle, non visualisable par définition. L'établissement d'un lien l'indépendance de la masse - uniquement à partir de l'observation des mouvements représente une approche didactique originale au regard de la littérature sur le sujet. La recherche de mise en images du contenu a ainsi permis de dégager un argument visuel pour étendre la notion de gravité à l'espace, argument qui pourra également être utilisé dans d'autres contextes.

61 La structure du raisonnement proposé constitue le squelette de la trame narrative de l'épisode. Nous montrons qu'il est possible de faire correspondre le fil conducteur du récit avec le cheminement conceptuel qui prévaut sur le propos. La scène d'exposition, l'élément déclencheur et la résolution du conflit sont consubstantiels de l'émergence des prérequis, du positionnement du problème à résoudre et de sa résolution. Cela implique des choix de mises en scène (transposition d'expériences de chutes libres dans le monde réel de jeunes adolescents tel que le permet le cadre d'une salle d'escalade), des choix de mises en situation (de personnages en train de jouer la comédie, de dialogues contradictoires) et de choix de mises en images (représentations visuelles des pensées des personnages, cadrage et point de vue du lecteur) adaptés aux contenus didactiques.

62 A contrario, la mise en scène en bande dessinée, et le format numérique par ailleurs, conditionnent la place des savoirs dans le récit et les possibilités de questionner ces savoirs. A cet égard, la proposition didactique centrale, basée sur l'indépendance de la masse et le changement de point de vue, a été choisie et approfondie pour l'apport potentiel des outils sémiotiques qu'offre le médium bande dessinée, telle que la représentation d'une scène de chute sous différents points de vue. Dans cet exemple, les auteurs ont mis à contribution les propriétés intrinsèques à la bande dessinée (séquençage d'une action), celles de la fiction (ralentissement du temps, moins adapté au documentaire graphique par exemple) et du format numérique (animation d'un décor 
accentuant le mouvement représenté). D’autres particularités du récit graphique telles que les images de pensée sont mises à profit pour justifier des analogies entre des environnement différents dont la juxtaposition, invraisemblable dans le monde réel, est acceptable dans le monde fictionnel.

Toutefois, bien que le profil des lecteurs ciblés - ou lecteur-modèle selon Eco (1985) - de cet épisode soit implicitement imaginé par les auteurs (élèves de cycle 4, lecteurs probables de bande dessinée et familiers de lectures sur écran, etc.), l'expérimentation de l'épisode, sous sa forme storyboardée, s'avère indispensable pour confronter les intentions initiales des auteurs avec la réception du public destinataire. Ceci est d'autant plus nécessaire que la bande dessinée est ici un support de lecture dépendant étroitement d'un accompagnement.

Les retours d'une première utilisation en classe permettent de dégager des pistes d'amélioration du storyboard et de son utilisation, en montrant en particulier l'importance d'un étayage sur les prérequis et d'un guidage de l'activité plus précis.

Malgré la difficulté à saisir les enjeux conceptuels, la grande majorité des élèves témoigne d'un sentiment de clarté et d'amusement pour le récit. Cette observation incite à questionner l'impact de l'amusement sur la perception d'éléments appartenants au raisonnement sous-jacent dans le scénario. Chez des élèves placés en position d'auteurs de planches de BD de sciences, l'humour est fréquemment mobilisé pour donner sens au récit mis en scène sous ce format narratif (De Hosson et al., dans le même numéro). Une prise en compte de ce paramètre, et plus largement de l'influence de la tonalité du récit sur la lecture de l'épisode vis-à-vis du raisonnement est nécessaire pour la suite de cette recherche.

Enfin cette première expérimentation s'est focalisée sur la compréhension par les élèves du cheminement conceptuel proposé, tel qu'il a été mis en scène dans la BD. Un point important pour une prochaine utilisation en classe sera de questionner également la manière dont les élèves s'approprient le problème initial tel qu'il est amené au début de l'épisode. 
Annexe 1, écrans 11-13
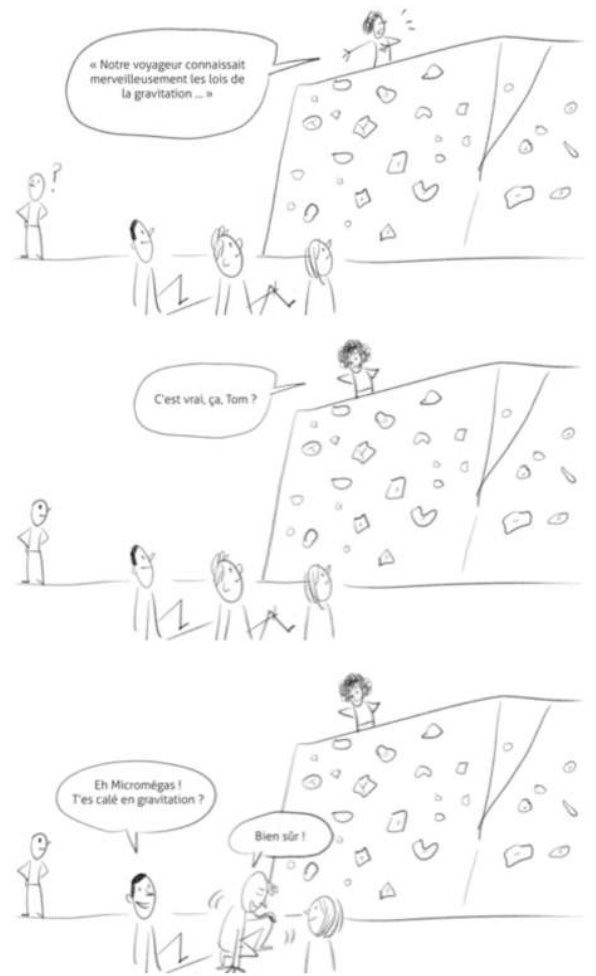

Govin et Bordenave, « tous droits réservés », 2019

Annexe 1-2, écrans 14-16
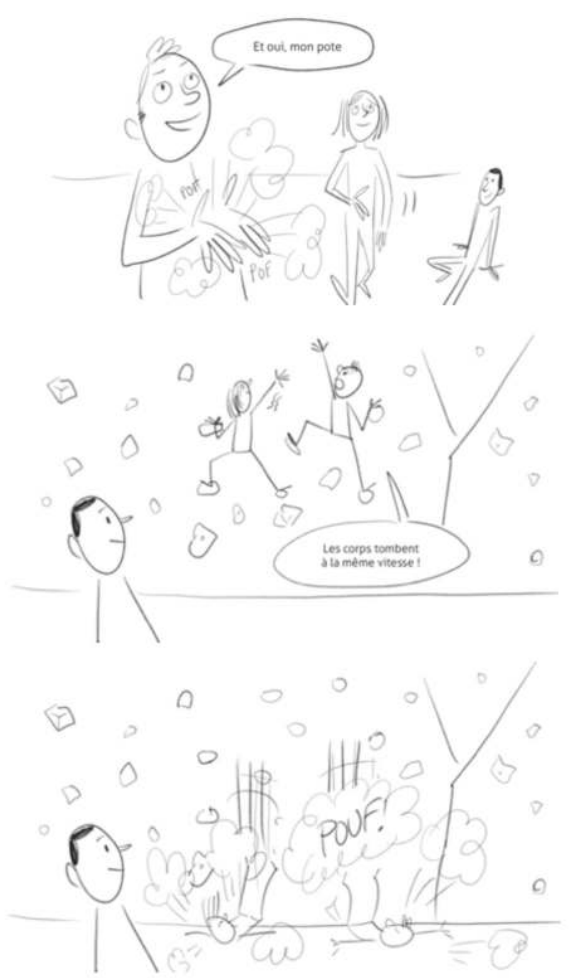

Govin et Bordenave, « tous droits réservés », 2019 
Annexe 1-3, écrans 17-19

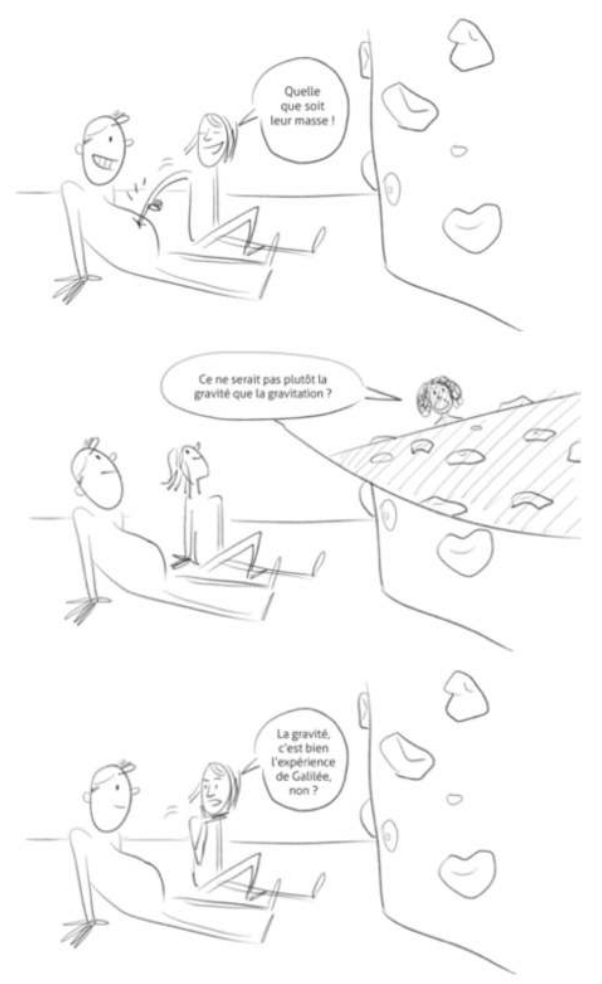

Govin et Bordenave, « tous droits réservés », 2019

Annexe 2, écrans 21-23
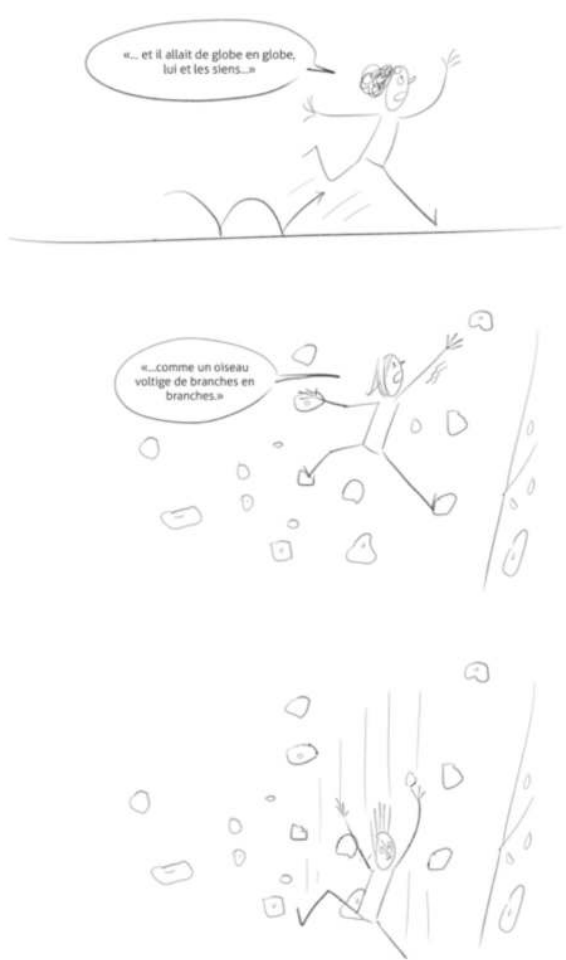

Govin et Bordenave, «tous droits réservés », 2019 
Co-construction et expérimentation d'une bande dessinée numérique pour la cla...

23

Annexe 2-2, écrans 24-26
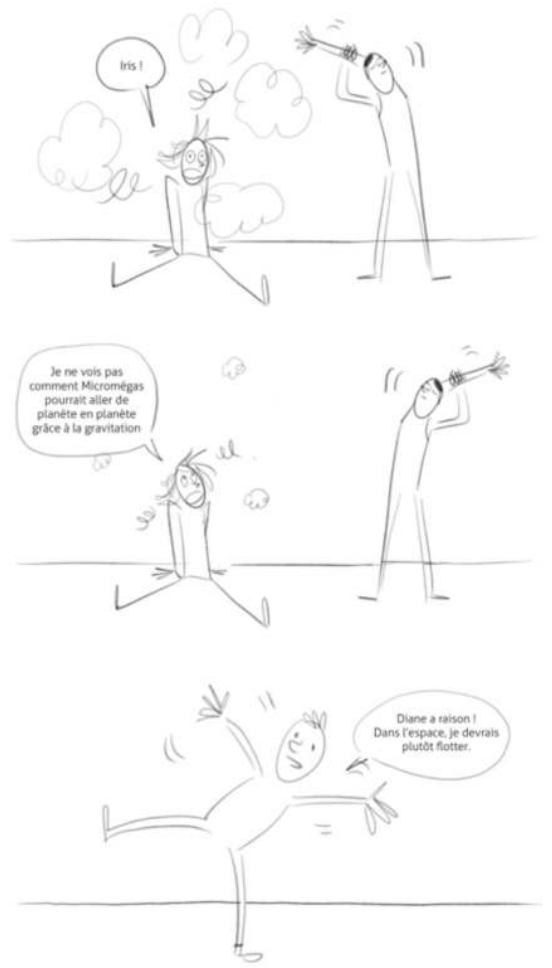

Govin et Bordenave, «tous droits réservés », 2019

Annexe 3, écrans 33-35
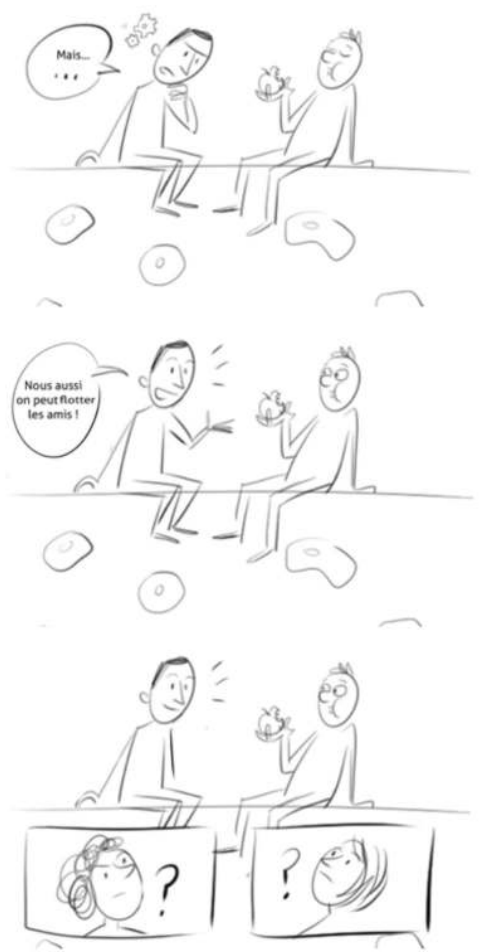

Govin et Bordenave, «tous droits réservés », 2019

Tréma, 51 | 2019 
Annexe 3-2, écrans 36-38
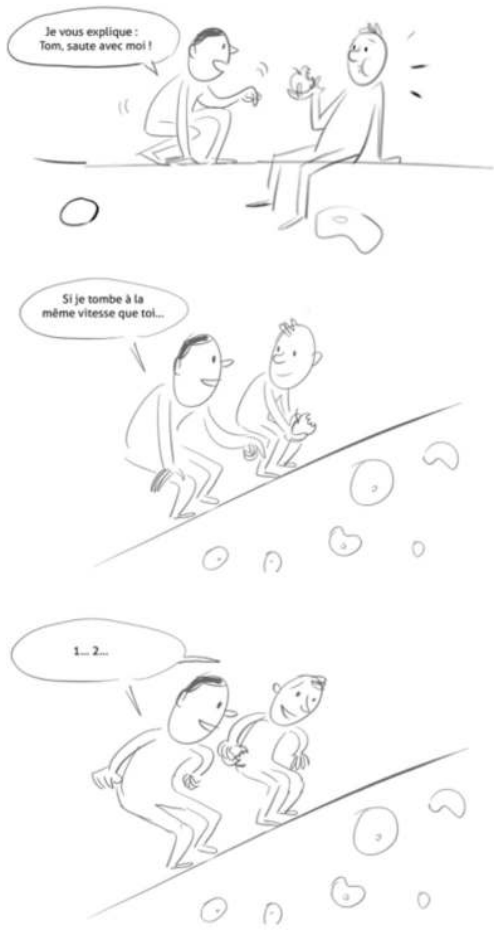

Govin et Bordenave, « tous droits réservés », 2019

Annexe 3-3, écrans 39-41

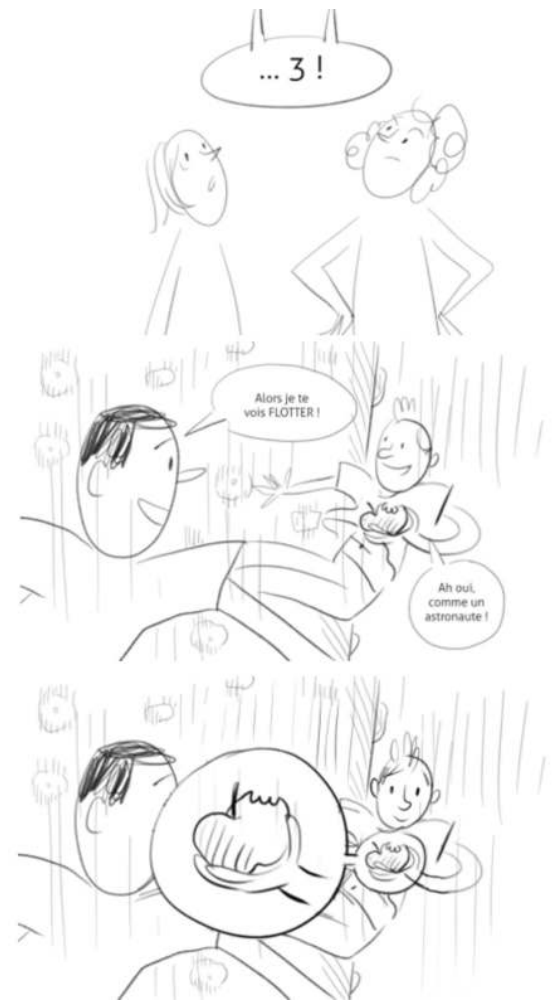

Govin et Bordenave, « tous droits réservés », 2019 
Annexe 3-4, écrans 42-44

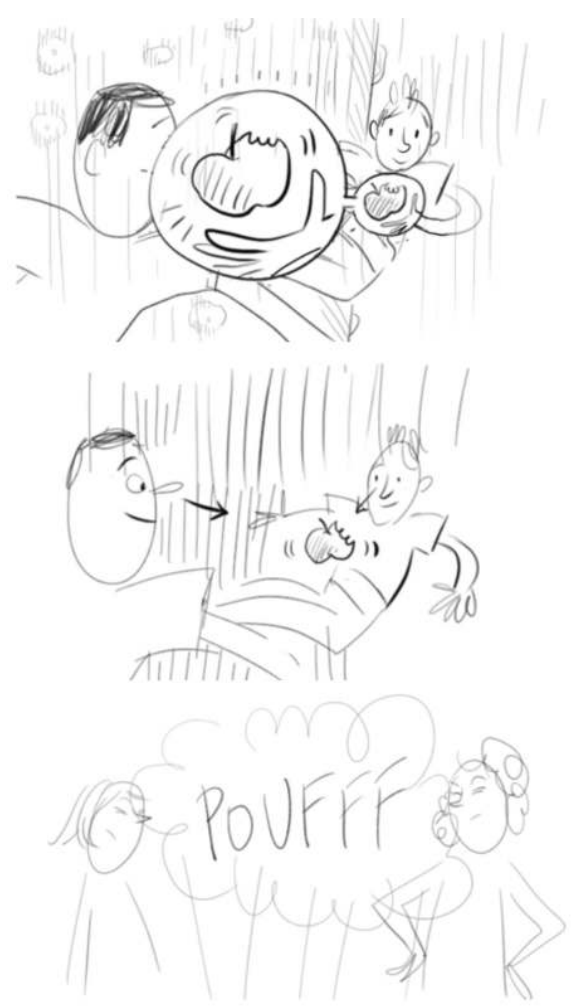

Govin et Bordenave, « tous droits réservés », 2019

Annexe 4, écrans 46-48

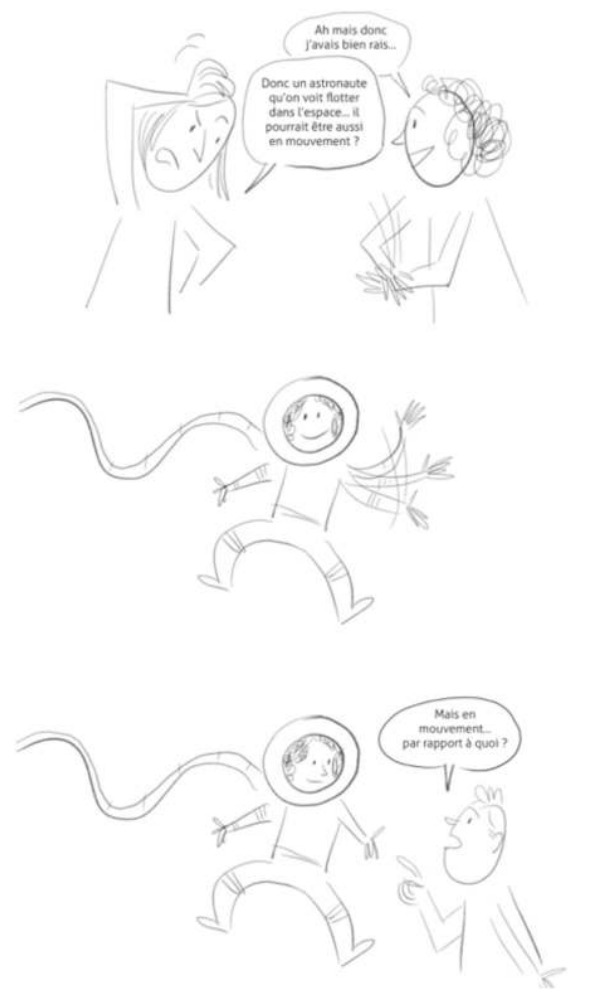

Govin et Bordenave, « tous droits réservés », 2019 
Annexe 4-2, écrans 49-51

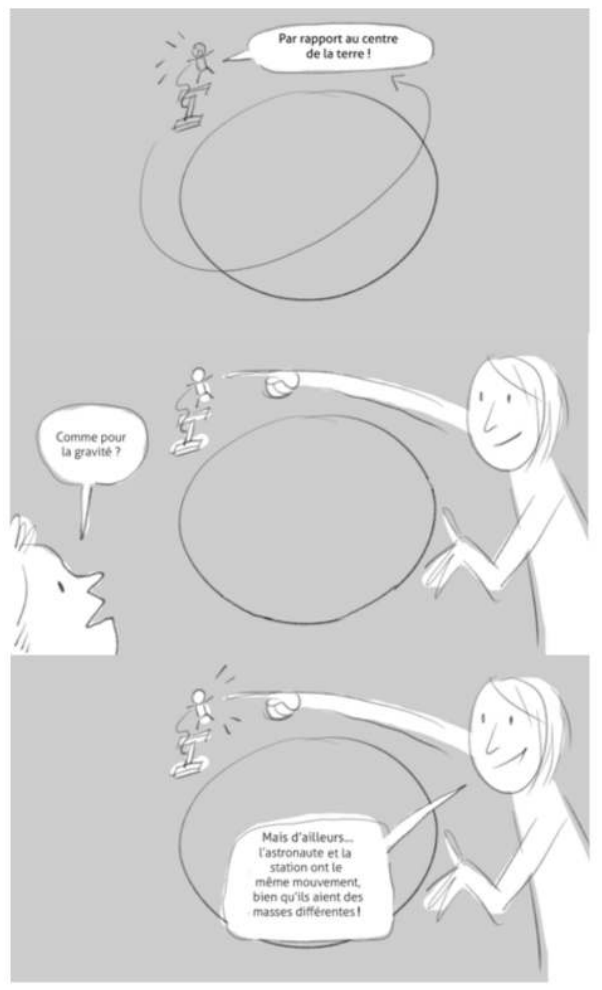

Govin et Bordenave, « tous droits réservés », 2019

Annexe 5, écrans 67-69
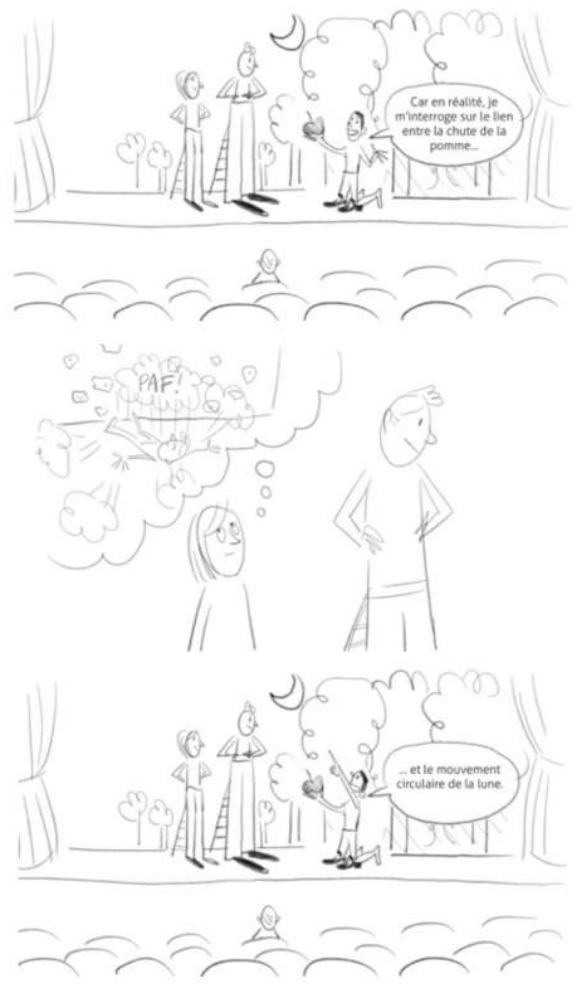

Govin et Bordenave, « tous droits réservés », 2019 
Annexe 5-2, écrans 70-72
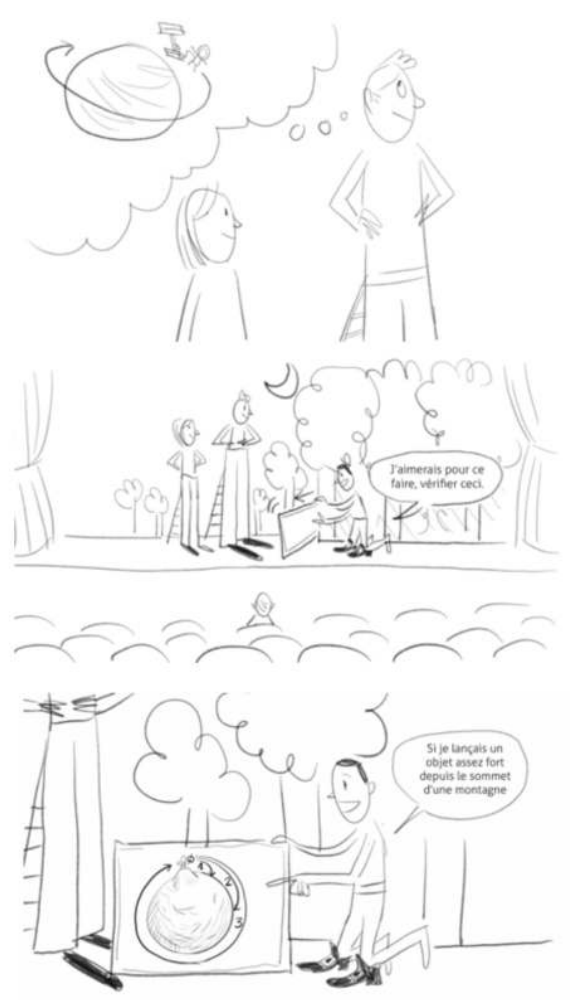

Govin et Bordenave, « tous droits réservés », 2019

Annexe 5-3, écrans 73-75
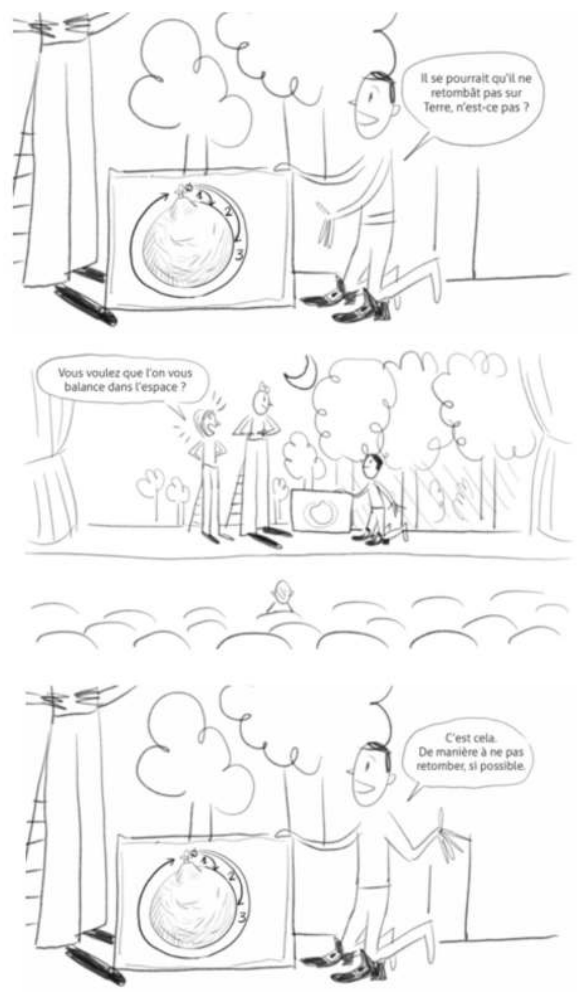

Govin et Bordenave, « tous droits réservés », 2019 
Annexe 5-4, écran 76
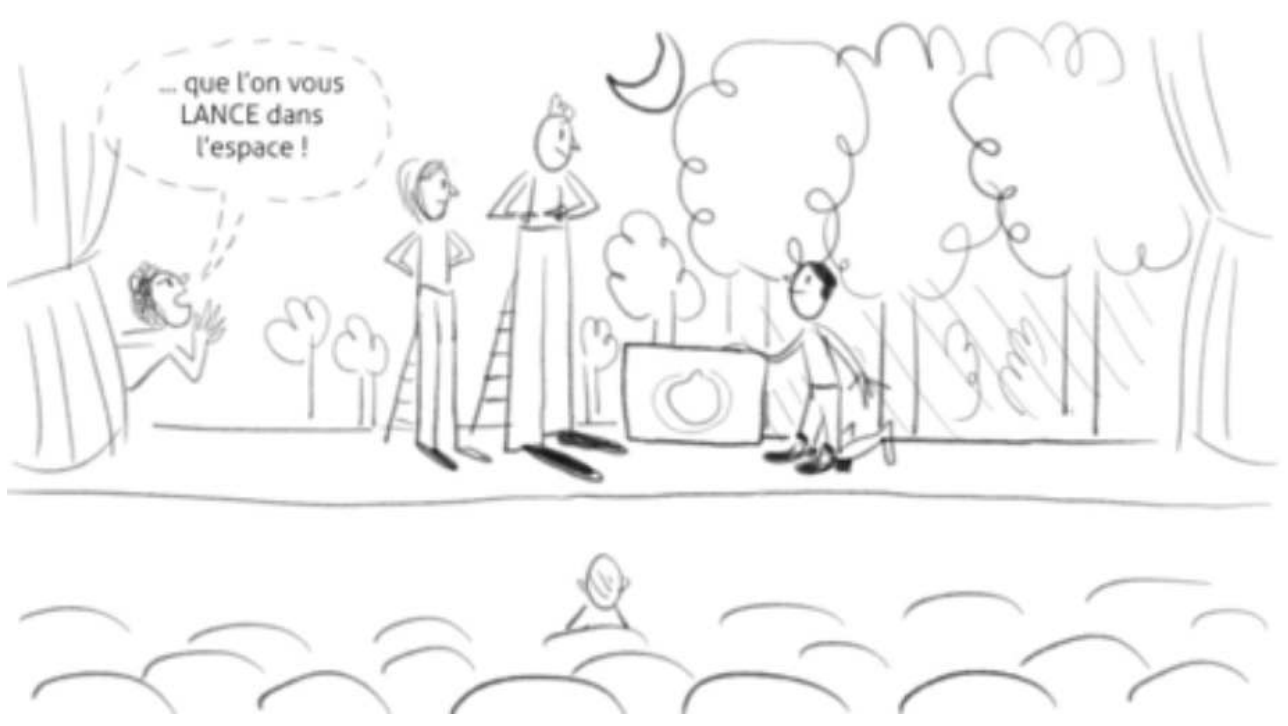

Govin et Bordenave, « tous droits réservés », 2019

Annexe 6, écran 80-83
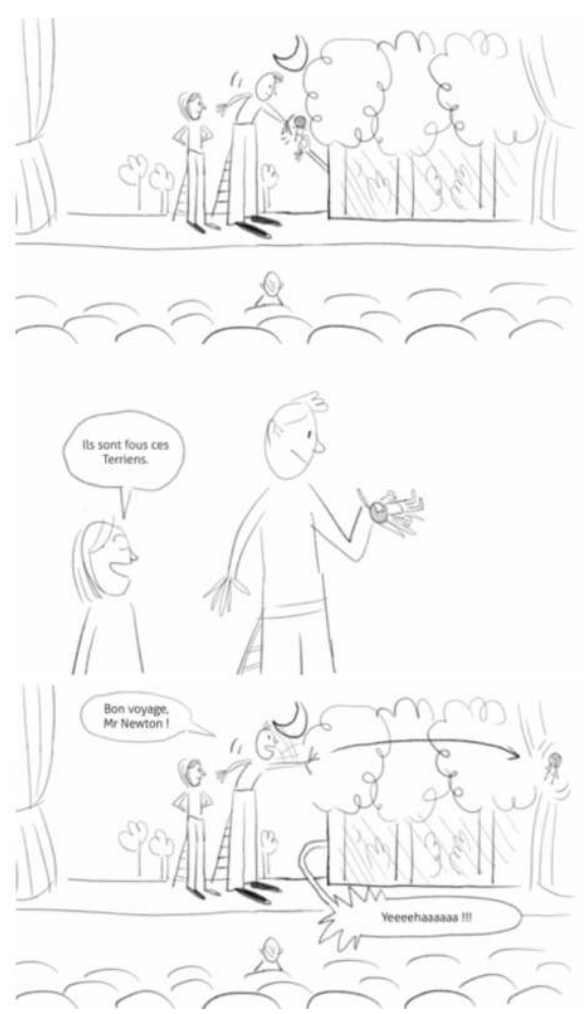

Govin et Bordenave, «tous droits réservés », 2019 
Annexe 6-2, écran 83-84
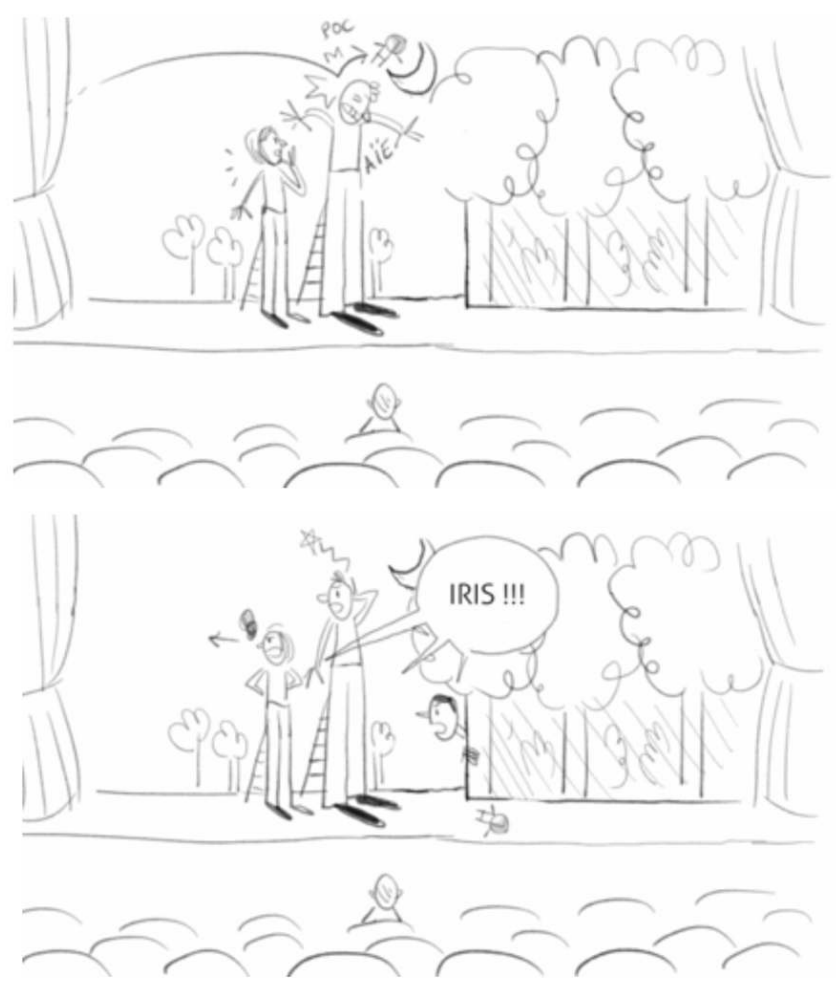

Govin et Bordenave, « tous droits réservés », 2019

Annexe 7 - Nouvelles propositions de questions pour une future expérimentation

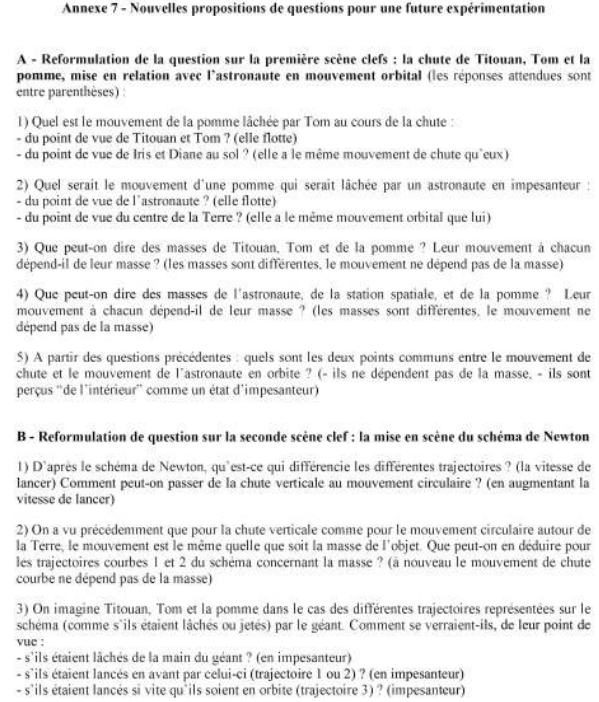




\section{BIBLIOGRAPHIE}

Arguel, P., Bonnefond, P., Matricon, J. (2017). ‘Lumière sur la BD’ : une approche modulaire de l'optique par l'analyse de vignettes issues de bandes dessinées. Rencontres Enseignement de l'Optique et Didactique (REOD). France : Limoges.

Audigier, F. \& Fillon, P. (1991). Enseigner l'histoire des sciences et des techniques. INRP.

Blanquet, E. (2016). De la science en bulles pour des situations de départ en démarche d'investigation à l'école primaire ? Colloque Telling Science, Drawing Science, 24-25 Nov. 2016, Angoulême : France

Bordenave, L. (2016). Les arcanes du récit de sciences en bande dessinée. Telling Science, Drawing Science, 23-24 nov. 2016, Angoulême : France.

Bordenave, L., Crépin-Obert, P. \& Pelé, M. (2016). Conception et analyse didactique d'une bande dessinée numérique sur l'histoire des sciences pour le collège : Les Grandiloquents. Telling Science, Drawing Science, 23-24 nov. 2016, Angoulême : France.

Chevallier, T. (2013). Tintin et la force gravitationnelle. Les cahiers pédagogiques, 506, 28-29.

Collver, J. \& Weitkamp, E. (2018). Alter Egos: An exploration of the motivations, perspectives, and identities of science comic creators. Journal of Science Communication, 17 (01) A01.

De Hosson C. (2016). Les “dialogues sur la manière dont se fait la vision" : genèse d'une saynète didactique d'inspiration historique. Telling Science, Drawing Science, 23-24 nov. 2016, Angoulême : France.

Durocher, E. (2016). Utiliser la Bande Dessinée en physique-chimie au collège. Telling Science, Drawing Science, 23-24 nov. 2016, Angoulême: France.

Eco U. (1985). Lector in fabula. Le rôle du lecteur. Paris : Grasset.

Eco U. (1996). Six promenades dans les bois du roman. Paris: Grasset.

Farinella, M. (2018). The potential of comics in science communication. Journal of Science Communication, 17 (01) Y01.

Hosler, J. \& Boomer, K.B. (2011). Are comic books an effective way to engage nonmajors in learning and appreciating science? CBE-Life Sciences Education, 10, 309-317.

Kavanagh, C. \& Sneider, C. (2006). Learning about Gravity II. Trajectories and Orbits: A Guide for Teachers and Curriculum Developers. Astronomy Education Review, 5(2), 53-102.

Maron, V. (2015). Une reconstruction conjointe de la dynamique et de la gravitation newtoniennes : conception et évaluation d'une séquence d'enseignement inspirée par l'histoire et l'épistémologie de la physique. Thèse de doctorat en didactique des sciences physiques. Université Paris Diderot et Sorbonne Paris Cité.

Peeters, B. (2002). Lire la bande dessinée. Paris : Flammarion.

Pelé, M. (2016). Problématisation autour de la circulation sanguine en classe de cinquième à partir d'une bande dessinée utilisant l'histoire des sciences. Mémoire de master de recherche en didactique des sciences expérimentales, Université Paris Diderot. 
Raux, H. (2018). Bande dessinée et diffusion des savoirs scientifiques : l'avènement du documentaire graphique? Rencontres Nationales de la Bande Dessinée 2 : Bande dessinée et éducation, Oct. 2017, Angoulême, France.

Triquet, E \& Bruguière R. (2014). Album de fiction, obstacles sur la métamorphose et propositions didactiques. Recherches en Didactique des Sciences et des Technologies (RDST), 9, 51-78.

Voltaire (2015). Micromégas, histoire philosophique. Collection Classico Lycée ( $\left.\mathrm{n}^{\circ} 117\right)$, Paris : Belin Gallimard.

\section{NOTES}

1. http://cache.media.eduscol.education.fr/file/programmes_2018/20/4/ Cycle_4_programme_consolide_1038204.pdf

2. Le terme « impesanteur » est préféré à celui d' " apesanteur », depuis un arrêté de 1995 relatif à la terminologie des sciences et techniques spatiales : «Le terme apesanteur est déconseillé pour éviter, dans le langage parlé, une confusion entre l'apesanteur et la pesanteur " (http:// www.legifrance.gouv.fr/affichTexte.do?cidTexte=JORFTEXT000000187248). Outre cette raison phonétique, le «a " privatif renforce l'association de l'état d' « apesanteur » à une absence de pesanteur, et ainsi par association : à l'absence de gravité dans l'espace.

3. ici une page est équivalente à un écran dans le texte de l'article

4. La question posée était : «La lecture de la BD que vous venez de lire vous a-t-elle plutôt : amusé $\square$ instruit $\square$ questionné $\square$ ennuyé ».

5. Avec comme items : « $\square$ pas vraiment $\square$ un peu $\square$ oui $\square$ oui beaucoup ».

6. On peut noter de plus que sur les 6 élèves indiquant s'être identifié à Titouan, il y a autant de garçons que de filles.

7. La question posée était : «Avez-vous trouvé l'activité proposée en binôme : $\square$ très facile facile $\square$ difficile $\square$ très difficile $\square$ ni trop facile, ni trop difficile ».

\section{RÉSUMÉS}

Cette étude porte sur la conception et l'expérimentation d'une bande dessinée numérique sur le thème de la gravitation au niveau collège, co-écrite par des auteurs de BD et un chercheur en didactique de la physique. La co-écriture a permis d'établir une correspondance entre les objectifs didactiques et le fil conducteur du récit, en tirant parti des spécificités du médium. Après une présentation du contexte de la $\mathrm{BD}$, la manière dont ont été articulés les choix didactiques, narratifs et graphiques sont discutées. Une première utilisation du storyboard a été expérimentée avec une classe de seconde générale et technologique au cours d'une séance. Les retours obtenus permettent d'identifier certains prérequis à la compréhension du raisonnement, des pistes d'amélioration du storyboard, et des recommandations concernant l'activité utilisant la bande dessinée comme support. Cette expérimentation ouvre également de nouvelles questions de recherche, dont celle de l'impact de l'humour sur la perception des éléments clefs du raisonnement. 
This study deals with the design and experimentation of a digital comic strip on the theme of gravitation for middle school level, co-constructed by comic book authors and a researcher in physics education research. The co-writing made it possible to establish a correspondence between the conceptual objectives and the common thread of the narrative, by taking advantage of the specificities of the medium. After presenting the context of the comic strip, we discuss the articulation of didactic, narrative and graphic choices. A first use of the storyboard has been experimented with a first year of high school class. The feedback obtained enable to identify certain prerequisites for understanding the content, ways of improving the storyboard, and recommendations for the activity based on the comic strip. This experimentation also opens new research questions for the future, including the impact of humor on the perception of the key elements of reasoning.

\section{INDEX}

Mots-clés : co-construction, bande dessinée, gravitation, histoire des sciences, expérimentation. Keywords : co-construction, comic strip, gravitation, history of sciences, experimentation.

\section{AUTEURS}

\section{VALENTIN MARON}

Laboratoire de Didactique André Revuz, Université Paris Diderot

\section{LAURENCE BORDENAVE}

Scénariste, Association Stimuli

\section{BARBARA GOVIN}

Illustratrice, Association Stimuli 